\title{
CONNECTED SIMPLE SYSTEMS, TRANSITION MATRICES AND HETEROCLINIC BIFURCATIONS
}

\author{
CHRISTOPHER MCCORD AND KONSTANTIN MISCHAIKOW
}

\begin{abstract}
Given invariant sets $A, B$, and $C$, and connecting orbits $A \rightarrow B$ and $B \rightarrow C$, we state very general conditions under which they bifurcate to produce an $A \rightarrow C$ connecting orbit. In particular, our theorem is applicable in settings for which one has an admissible semiflow on an isolating neighborhood of the invariant sets and the connecting orbits, and for which the Conley index of the invariant sets is the same as that of a hyperbolic critical point. Our proof depends on the connected simple system associated with the Conley index for isolated invariant sets. Furthermore, we show how this change in connected simple systems can be associated with transition matrices, and hence, connection matrices. This leads to some simple examples in which the nonuniqueness of the connection matrix can be uxplained by changes in the connected simple system.
\end{abstract}

\section{INTRODUCTION}

One of the most important and challenging problems in the study of dynamical systems is the question of the existence of global bifurcations. The appearance or disappearance of heteroclinic orbits, i.e., trajectories which limit in forward and backward time to critical points, provide the simplest example of such bifurcations. Clearly what makes detecting such orbits difficult is the fact that local analysis of the problem is of limited use. Thus, in order to study this problem on a general level, one is naturally led to using topological techniques. In particular, the usefulness of the Conley index in this capacity has already been demonstrated. In most applications, a weak form of this index has been used, either the homotopy type of the index or the homology of the index. However, there exists a much stronger version of the Conley index, namely as a connected simple system. This version uses not only the homotopy type of a pointed space, but also the homotopy types of a collection of flow defined maps. This turns out to be a very precise index and, as shall be demonstrated in this paper, it can be used to prove the bifurcation and persistence of heteroclinic orbits under fairly weak assumptions.

Received by the editors April 20, 1989 and, in revised form, June 19, 1990.

1980 Mathematics Subject Classification (1985 Revision). Primary 34C35, 58F14.

Key words and phrases. Bifurcations, Conley index, connection matrices, connected simple systems, heteroclinic orbits, transition matrices.

The first author was supported in part by DARPA Applied and Computational Mathematics Program.

The second author was supported in part by an AURIG from Michigan State University and the National Science Foundation. 


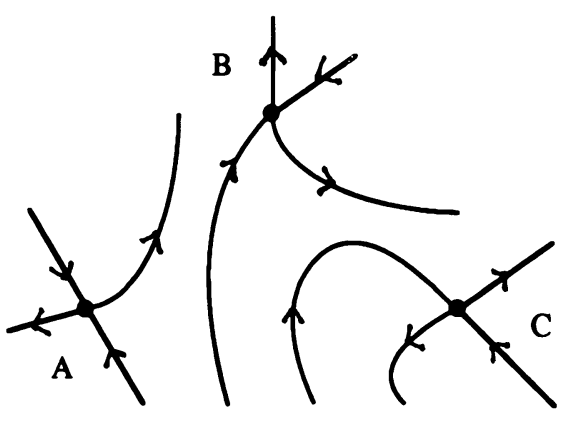

$\lambda_{0}$

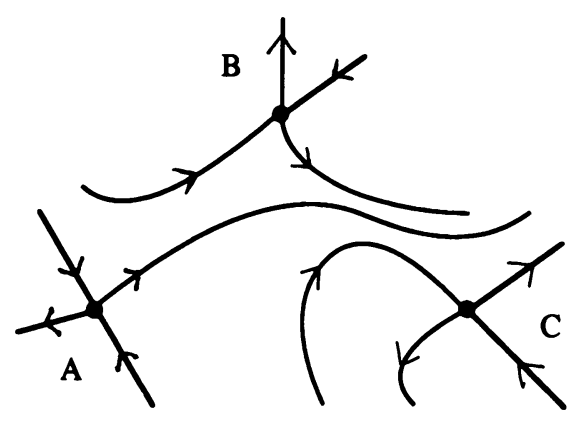

$\lambda_{3}$

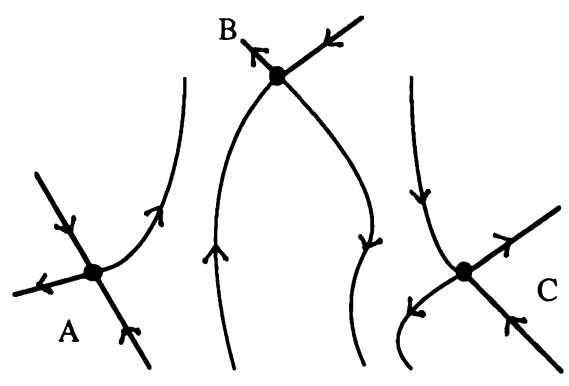

$\lambda_{1}$

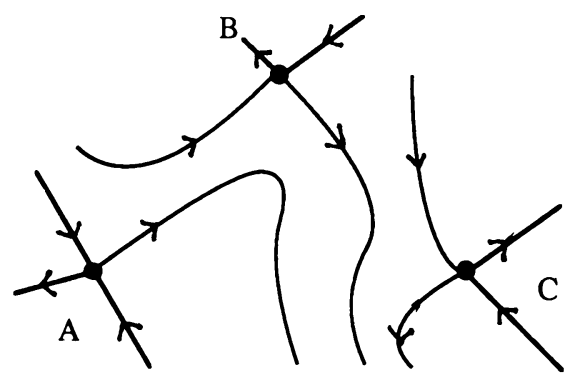

$\lambda_{2}$

FIGURE 1

At the same time, there is a problem with the connected simple system index: it does not lend itself well to computation. Another idea due to Conley is that of the connection matrix. These matrices are based on the homology groups associated to the Conley index and as such are relatively easy to generate and to work with. However, they suffer from an inherent lack of uniqueness. Our understanding of this nonuniqueness is rather limited. In some examples it has been related to the existence of global bifurcations, and hence, is an important feature of connection matrices. Unfortunately, it is not known whether all the nonuniqueness is related to some form of dynamics or whether it is an algebraic artifice which appears because of the purely algebraic construction of connection matrices. Our hope is that this nonuniqueness can be explained by using the index theory on the level of connected simple systems. This in turn could lead to a simplification of the problems of computing with connected simple systems.

The modest goal of this paper is to consider these questions in a setting in which the Conley index of each Morse set is a pointed $n$-sphere. Thus the correspondence between the homotopy level of the theory (connected simple systems) and the homology level of the theory (connection matrices) is made simple via the Hurewicz isomorphism theorem. A concrete description of the type of problem which motivated this study is as follows. Consider a continuous 2-parameter family of flows in $\mathbf{R}^{2}$. Letting $\Lambda$ denote this parameter space, choose $\lambda_{i} \in \Lambda, i=0,1,2$, and 3 and assume that the phase portrait at each 


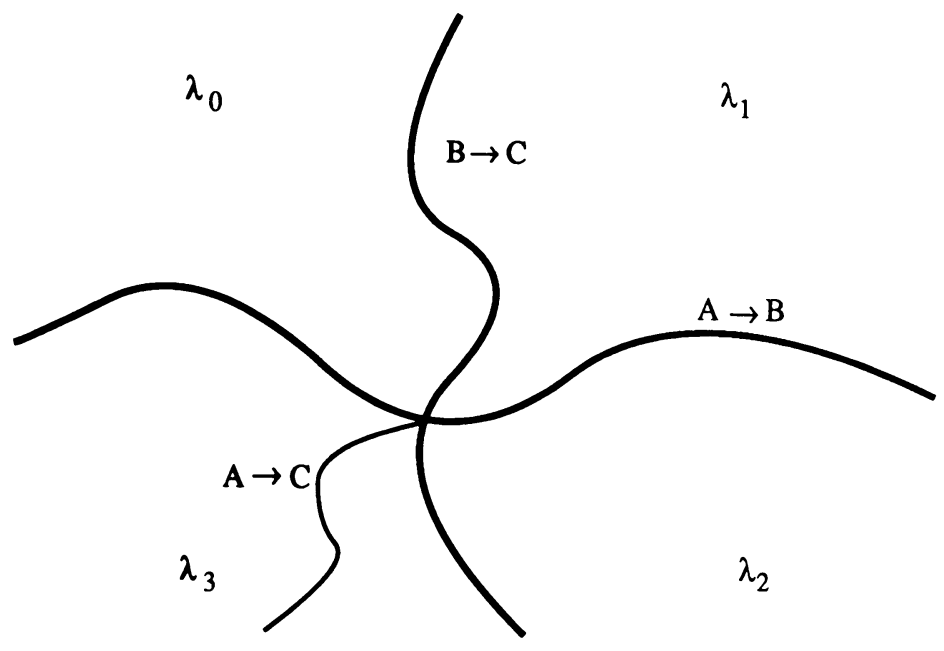

FIGURE 2

of these parameter values is as shown in Figure 1. Notice that in each diagram there are 3 critical points. In the language of Conley, these are Morse sets each with index $\Sigma^{1}$, i.e. pointed 1-spheres. By inspection one notices that to get from the phase portrait associated with $\lambda_{0}$ to that associated with $\lambda_{1}$ (and similarly from $\lambda_{2}$ to $\lambda_{3}$ ) a heteroclinic orbit from $B \rightarrow C$ must have occurred. Again by inspection, going from $\lambda_{0}$ to $\lambda_{3}$ and $\lambda_{1}$ to $\lambda_{2}$ entails an $A \rightarrow B$ heteroclinic orbit occurring. This is indicated in Figure 2, by the heavy lines. However, closer inspection of these diagrams leads one to the conclusion that there, also, must be a family of parameter values along which an $A \rightarrow C$ heteroclinic orbit also occurs. Again, this is indicated in Figure 2.

Results of this nature are by now well established (see [K] and references therein). In particular our work was motivated by the following theorem of Chow, Deng and Terman [CDT]. Consider the differential equation $x^{\prime}=$ $f(x, \mu)$ where $x \in \mathbf{R}^{m+n}$ and $\mu=\left(\mu_{1}, \mu_{2}\right) \in \mathbf{R}^{2}$. Assume that

(H1) $f \in C^{2}\left(\mathbf{R}^{m+n} \times \mathbf{R}^{2}, \mathbf{R}^{m+n} \times \mathbf{R}^{2}\right)$.

(H2) There exist $A, B, C \in \mathbf{R}^{m+n}$ such that $f(A, \mu)=f(B, \mu)=f(C, \mu)$ $=0$ for all $\mu$. Here $A \neq B \neq C$ but it is possible that $A=C$.

(H3) When $\mu=O=(0,0)$, there exist connections $\Gamma_{B C}$ from $B$ to $C$ and $\Gamma_{A B}$ from $A$ to $B$.

(H4) For $\alpha \in\{A, B, C\}$ and all $\mu, \alpha$ is a hyperbolic rest point with an $m$-dimensional stable manifold $W_{\alpha}^{s}(\mu)$ and $n$-dimensional unstable manifold $W_{\alpha}^{u}(\mu)$.

(H5) Let $T_{p} M$ be the tangent space of manifold $M$ at point $p$. Then

$$
\operatorname{dim}\left(T_{p} W_{A}^{u}(O) \cap T_{p} W_{B}^{s}(O)\right)=1
$$

and

$$
\operatorname{dim}\left(T_{q} W_{B}^{u}(O) \cap T_{q} W_{C}^{s}(O)\right)=1
$$

for all $p \in \Gamma_{A B}$ and $q \in \Gamma_{B C}$.

(H6) $W_{A}^{u}\left(0, \mu_{2}\right)$ and $W_{B}^{s}\left(0, \mu_{2}\right)$ cross transversely as $\mu_{2}$ crosses 0 , and $W_{B}^{u}\left(\mu_{1}, 0\right)$ and $W_{C}^{s}\left(\mu_{1}, 0\right)$ cross transversely as $\mu_{1}$ crosses 0. 
Theorem 1.1. Assume (H1)-(H6). There exists $\rho>0$ such that if $|\gamma|<\rho$, then there exists $\mu_{1}(\gamma)$ and $\mu_{2}(\gamma)$ such that at $\mu=\left(\gamma, \mu_{2}(\gamma)\right)$ there is a connection from $A$ to $B$, and at $\mu=\left(\mu_{1}(\gamma), \gamma\right)$ there is a connection from $B$ to $C$. Moreover,

$$
\lim _{\gamma \rightarrow 0} \mu_{1}(\gamma)=\lim _{\gamma \rightarrow 0} \mu_{2}(\gamma)=0 .
$$

Theorem 1.2. Assume (H1)-(H6), and that there exist curves $\Gamma_{A B}$ and $\Gamma_{B C}$ in $\mathbf{R}^{2}$ which intersect transversely at $O$ such that there exists a connection from $A$ to $B$ for every $\mu \in \Gamma_{A B}$ and a connection from $B$ to $C$ for every $\mu \in \Gamma_{B C}$. Then there exists a continuous map $g:(0,1] \rightarrow \mathbf{R}^{2}$ such that for each $s \in(0,1]$, there exists a connection from $A$ to $C$ at $\mu=g(s)$. If $s_{1} \neq s_{2}$, then the corresponding connections are not the same. Finally, $\lim _{s \rightarrow 0} g(s)=O$.

Though these are obviously important results, one is quickly led to question the necessity of the hypothesis.

(H1) Consider the example of the planar problem described in Figures 1 and 2. The intuitive arguments which lead to the existence of the heteroclinic orbits $A \rightarrow B, B \rightarrow C$, and $A \rightarrow C$, do not involve the smoothness of the vector field. Thus one would conjecture that if $x^{\prime}=f(x, \mu)$ leads to a continuous parameterized family of flows then the same results should hold. In particular, it should not be necessary that $f \in C^{2}$.

(H2) The reason that the $A \rightarrow B, B \rightarrow C$, and $A \rightarrow C$ orbits occur is because the stable and unstable manifolds of the invariant sets have to get "past" one another. In particular, the structure of the invariant set does not appear to be of any importance. What matters is the structure of the unstable set corresponding to the invariant sets.

(H3) This assumption is essential if one wishes to demonstrate the existence of an $A \rightarrow C$ connecting orbit.

(H4) The same argument as for (H2) suggests that this can be weakened. Furthermore, the importance of the dimension and structure of the stable manifold is questionable.

(H5)-(H6) These assumptions, while "generic," are in general very difficult to verify. Once again, what appears to be important in the simple example of Figure 1 is that the stable and unstable manifolds cross, not how they cross.

These concerns are what motivated our study of this problem. In particular, it is clear that to address these comments the problem needs to be approached from a purely topological point of view. Thus in $\S 2$, we shall review the definitions and concepts related to the Conley index and connected simple systems. In the setting of a flow on a locally compact space references for this material are Conley [C], Kurland [Ku1-Ku3], and Salamon [S]. However, since our results are as easily obtained for admissible semiflows on arbitrary metric spaces, and since this setting minimizes the importance of the stable manifold, we shall adopt this later point of view. In this case, the reader is referred to Rybakowski [Ry]. With this background, we proceed in $\S 3$ to develop a method for detecting bifurcations using connected simple systems. Then, in $\S 4$ we prove our version of the global bifurcation theorems for heteroclinic orbits.

Of course, at this point we have only completed half of our stated goal. In particular, we have not related this work on connected simple systems to the theory of the connection matrix. Thus, in $\S 5$ we review the algebraic theory of the connection matrix and describe transition systems. Finally, in $\S 6$ we describe 
how, in some cases, maps between connected simple systems correspond to conjugations of connection matrices, and so generate transition matrices. While the results of this section are preliminary, they indicate an approach to one of the central questions in the connection matrix theory.

As mentioned above, an inherent property of connection matrices is their nonuniqueness. In particular, the continuation theorem for connection matrices states that the set of connection matrices at a given parameter value contains the set of connection matrices for all nearby parameter values. Thus, at parameter values where bifurcations occur, one would expect nonuniqueness of connection matrices. This raises two questions: Is this the only source of nonuniqueness; and does nonuniqueness occur at all bifurcation points? The answer to the first question is now known and the second is definitely false.

Our results, however, indicate that in the limited setting we consider, the methods developed in $\S 3$ generate systems of matrices which are not the identity if and only if a bifurcation occurs, and which generate connection matrices via a homology functor from the homotopy category. This indicates that the failure of connection matrices to detect bifurcations might be quantified in terms of this functor.

\section{The CONLEY INDEX}

The results of this paper are applicable for any dynamical system on a metric space $X$ which is induced by a local semiflow. To be more precise, let $D$ be an open set in $X \times[0, \infty)$ and $\varphi: D \rightarrow X$ be a continuous map which will be denoted by $\varphi(x, t)=x \cdot t . \varphi$ is a local semiflow if:

(1) For every $x \in X$ there exists $\omega_{x} \in(0, \infty]$ such that $(x, t) \in D$ if and only if $t \in\left[0, \omega_{x}\right)$,

(2) $x \cdot 0=x$,

(3) if $(x, t),(x \cdot t, s) \in D$, then $(x, t+s) \in D$ and $x \cdot(t+s)=(x \cdot t) \cdot s$. A solution through $x \in X$, is a continuous map $\sigma_{x}:\left(a, \omega_{x}\right) \rightarrow X$ where $a \in[-\infty, 0)$ satisfying

(1) $\sigma_{x}(0)=x$,

(2) for $t \in\left(a, \omega_{x}\right)$ and $s>0$ with $s+t<\omega_{x}$, it follows that $\sigma_{x}(t) \cdot s=$ $\sigma_{x}(t+s)$.

If $a=-\infty$, then $\sigma_{x}$ is called a full left solution. If, in addition, $\omega_{x}=\infty$, then $\sigma_{x}$ is referred to as a full solution.

If $N \subset X$ and $J \subset[0, \infty)$, then define $N \cdot J=\{x \cdot t \mid x \in N$ and $t \in J\}$. If for each $x \in N, \omega_{x}=\infty$, then the $\omega$-limit set of $N$ is given by $\omega(N)=\bigcap_{t>0} \operatorname{cl}(N \cdot[t, \infty))$. If $\sigma_{x}$ is a full left solution, then we define the $\omega^{*}$-limit set of $\sigma_{x}$ to be the set $\omega^{*}\left(\sigma_{x}\right)=\bigcap_{t>0} \operatorname{cl}\left(\sigma_{x}((-\infty, t])\right)$. Note that since $\sigma_{x}$ is not uniquely determined for $x, \omega^{*}\left(\sigma_{x}\right)$ depends on the full left solution chosen, not just $x$. Let

$$
\begin{aligned}
& A^{+}(N)=\left\{x \in X \mid x \cdot\left[0, \omega_{x}\right) \subset N\right\} \text { and } \\
& A^{-}(N)=\left\{x \in X \mid \exists \text { a full left solution } \sigma_{x} \text { such that } \sigma_{x}((-\infty, 0]) \subset N\right\} .
\end{aligned}
$$

A set $S$ is called invariant if $S=A^{+}(S)=A^{-}(S)$. It is implicit in this definition that there is a full solution through each point $x \in S$. Notice that $S=A(N) \equiv A^{+}(N) \cap A^{-}(N)$ is an invariant set. If, in addition $S \subset \operatorname{int}(N)$, then $S$ is called an isolated invariant set and $N$ is an isolating neighborhood of $S$. 
$N \subset X$ is called admissible provided that

(1) given any two sequences $\left\{x_{n}\right\} \subset N$ and $\left\{t_{n}\right\} \subset[0, \infty)$ satisfying $t_{n} \rightarrow \infty$ and $x_{n} \cdot\left[0, t_{n}\right] \subset N$, then the sequence $\left\{x_{n} \cdot t_{n}\right\}$ is precompact,

(2) if $x \in N$ and $\omega_{x}<\infty$, then $x \cdot\left[0, \omega_{x}\right) \not \subset N$.

Definition 2.1. Let $S$ be an isolated invariant set. An index pair for $S$ is an ordered pair of closed sets $(N, L)$ with $L \subset N$ such that

(a) $S \subset \operatorname{int}(N \backslash L)$ and $\operatorname{cl}(N \backslash L)$ is an isolated neighborhood for $S$.

(b) $L$ is positively invariant in $N$, i.e., given $x \in L$ and $x \cdot[0, t] \subset N$, then $x \cdot[0, t] \subset L$.

(c) $L$ is an exit-set for $N$, i.e., given $x \in N$ and $t_{1}>0$ such that $x \cdot t_{1} \neq N$, there exists $t_{0} \in\left[0, t_{1}\right] \subset L$ and $x \cdot\left[0, t_{0}\right] \subset N$.

As the name indicates, index pairs are used to define the Conley index of the isolated invariant set $S$. Before giving the definition, however, we digress to introduce some necessary terminology and notation. Given closed sets $L \subset N$, let

$$
N / L=((N \backslash L) \cup[L],[L])
$$

be the pointed space which is obtained by collapsing $L$ to a single point denoted by $[L]$. The topology on $N / L$ is given by defining $U \subset N \backslash L$ to be open if either $U$ is open in $N$ and $U \cap L=\varnothing$, or the set $(U \cap(N / L)) \cup L$ is open in $N$. Let $\left(Y, y_{0}\right)$ and $\left(Z, z_{0}\right)$ be pointed spaces. [ $\left.Y ; Z\right]$ denotes the set of homotopy classes of continuous base point preserving maps from $Y$ to $Z$ and $[f]$ denotes the homotopy class of the continuous base point preserving map $f: Y \rightarrow Z$. If $\left(Y, y_{0}\right)$ and $\left(Z, z_{0}\right)$ are homotopic via base point preserving maps, then we write $Y \sim Z$. The sum of $\left(Y, y_{0}\right)$ and $\left(Z, z_{0}\right)$ is written as

$$
Y \vee Z=Y \times\left\{z_{0}\right\} \cup\left\{y_{0}\right\} \times Z \subset Y \times Z \text {. }
$$

Definition 2.2. A connected simple system (CSS) consists of a collection $I_{o}$ of pointed spaces along with a collection $I_{m}$ of homotopy classes of maps between these such that

(1) $\operatorname{hom}\left(Z, Z^{\prime}\right)=\left\{[f] \in\left[Z ; Z^{\prime}\right] \mid[f] \in I_{m}\right\}$ is nonempty and consists of a single element for each ordered pair $Z, Z^{\prime}$ of spaces in $I_{o}$,

(2) if $Z, Z^{\prime}, Z^{\prime \prime} \in I_{o},[f] \in \operatorname{hom}\left(Z, Z^{\prime}\right)$, and $\left[f^{\prime}\right] \in \operatorname{hom}\left(Z^{\prime}, Z^{\prime \prime}\right)$, then $\left[f^{\prime} \circ f\right] \in \operatorname{hom}\left(Z, Z^{\prime \prime}\right)$.

(3) $\operatorname{hom}(Z, Z)=\left\{\left[1_{Z}\right]\right\}$ for all $Z \in I_{0}$.

Definition 2.3. A CSS morphism $\Phi: I \rightarrow J$ between the connected simple systems $I=\left(I_{o}, I_{m}\right)$ and $J=\left(J_{o}, J_{m}\right)$ is a collection of homotopy classes of maps between spaces in $I_{o}$ and spaces in $J_{o}$ such that

(1) for every $Y \in I_{o}$ and $Z \in J_{o}$, the set $\Phi(Y, Z)=\{[\phi] \in[Y ; Z] \mid[\phi] \epsilon$ $\Phi\}$ contains a unique element,

(2) if $Y, Y^{\prime} \in I_{o}$ and $Z, Z^{\prime} \in J_{o}$, and if $[\phi] \in \Phi(Y, Z),[f] \in \operatorname{hom}\left(Y^{\prime}, Y\right)$, $[g] \in \operatorname{hom}\left(Z, Z^{\prime}\right)$, then $[g \circ \phi \circ f] \in \Phi\left(Y^{\prime}, Z^{\prime}\right)$.

We are finally in the position to define the Conley index.

Definition 2.4. Let $S$ be an isolated invariant set. The Conley index of $S$ is the connected simple system $I(S)=\left(I_{o}(S), I_{m}(S)\right)$, where

$I_{o}(S)=\{N / L \mid(N, L)$ is an index pair of $S\}$.

$I_{m}(S)=\left\{[f] \mid f: N / L \rightarrow N^{\prime} / L^{\prime}\right.$ is a flow defined map between any two index pairs $(N, L)$ and $\left(N^{\prime}, L^{\prime}\right)$ of $\left.S\right\}$. 


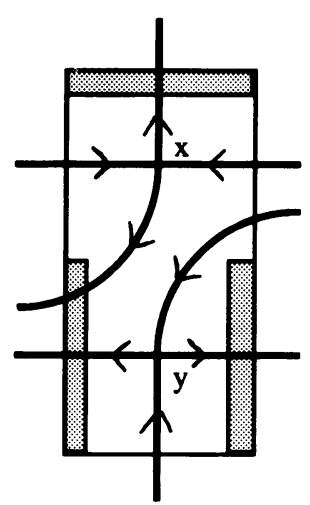

$S_{a}$

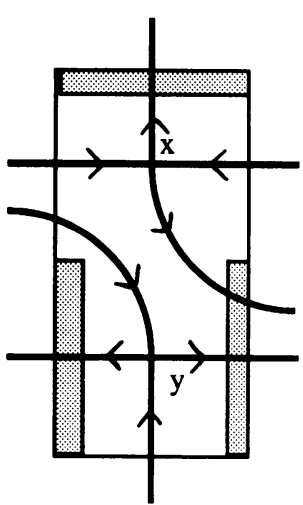

$\mathrm{S}_{\mathrm{b}}$

FIGURE 3

Stated this way it is not at all clear that the Conley index is a well defined concept. However, in the context of flows on locally compact spaces this follows from [S, Theorem 4.10] where the following explicit forms for the flow defined maps $f$ are given. Let $(N, L)$ and $\left(N^{\prime}, L^{\prime}\right)$ be index pairs for $S$ then for $t$ sufficiently large define $f: N / L \rightarrow N^{\prime} / L^{\prime}$ by

$$
f([x])= \begin{cases}{[x \cdot 3 t]} & \text { if } x \cdot[0,2 t] \subset N \backslash L, x \cdot[t, 3 t] \subset N^{\prime} \backslash L^{\prime} \\ {\left[L^{\prime}\right]} & \text { otherwise }\end{cases}
$$

In our context of semiflows on metric spaces we refer the reader to [Ry, Theorem 9.1].

A simple observation is that given $S$ with index pairs $(N, L)$ and $\left(N^{\prime}, L^{\prime}\right)$, $N / L \sim N^{\prime} / L^{\prime}$. Thus one can define the homology Conley index by

$$
C H_{*}(S)=H_{*}(N / L,[L]) \text {. }
$$

Before continuing with this brief review of the theory, consider the following simple example which illustrates the difference between the connected simple system index, $I(S)$, and the homology index $C H_{*}(S)$. Figure 3 contains two isolated invariant sets, $S_{a}$ and $S_{b}$, each consisting of two critical points labelled $x$ and $y$. Index pairs $(N, L)$ for these invariant sets are also indicated where $L$ consists of the shaded regions. Clearly, $C H_{*}\left(S_{a}\right) \approx H_{*}(N, L) \approx C H_{*}\left(S_{b}\right)$. However, it is intuitively clear that $I\left(S_{a}\right)$ and $I\left(S_{b}\right)$ differ since the unstable manifold of the critical point $x$ defines a different generator for $C H_{*}\left(S_{a}\right)$ than it does for $C H_{*}\left(S_{b}\right)$. The precise sense in which $I\left(S_{a}\right)$ and $I\left(S_{b}\right)$ differ will be developed in $\S 3$.

We now wish to turn to the question of how does one decompose an isolated invariant set $S$. However, we need to begin with a discussion of partially ordered sets. A partially ordered set, $(P,>)$, consists of a finite set $P$ along with a strict partial order relation, $>$, which satisfies:

(i) $p>p$ never holds for $p \in P$, and

(ii) if $p>p^{\prime}$ and $p^{\prime}>p^{\prime \prime}$, then $p>p^{\prime \prime}$ for all $p, p^{\prime}, p^{\prime \prime} \in P$.

An interval in $(P,>)$ is a subset, $I \subset P$, such that given $p, p^{\prime} \in I$, if $p>p^{\prime \prime}>$ $p^{\prime}$ then $p^{\prime \prime} \in I$. The set of intervals in $(P,>)$ is denoted by $\mathscr{I}(P,>)$ or just $\mathscr{F}$ if $(P,>)$ is fixed. An attracting interval, $I \in \mathscr{I}$, satisfies the additional 
condition that if $p \in \mathscr{I}$ and $p>p^{\prime}$, then $p^{\prime} \in I . \mathscr{A}=\mathscr{A}(P,>)$ denotes the set of attracting intervals. An adjacent $n$-tuple of intervals in $(P,>)$ is an ordered collection $\left(I_{1}, \ldots, I_{n}\right)$ of mutually disjoint intervals satisfying

(i) $\bigcup_{i=1}^{n} I_{i} \in \mathscr{I}$,

(ii) $p \in I_{i}, p^{\prime} \in I_{j}$, and $i<j$ imply $p \ngtr p^{\prime}$.

The collection of adjacent $n$-tuples is denoted by $\mathscr{I}_{n}(P,>)=\mathscr{I}_{n}$. If $(I, J)$ is an adjacent pair (i.e., a 2-tuple) of intervals, then we set $I J=I \cup J . I$ and $J$ are said to be noncomparable if both $(I, J)$ and $(J, I)$ belong to $\mathscr{I}_{2}$. Finally, if $\left\{p, p^{\prime}\right\} \in \mathscr{I}$, then $p$ and $p^{\prime}$ are said to be adjacent.

Let $C\left(M(p), M\left(p^{\prime}\right)\right)=\left\{x \mid \omega(x) \subset M\left(p^{\prime}\right)\right.$ and $\exists$ a full left solution $\sigma_{x}$ such that $\left.\omega^{*}\left(\sigma_{x}\right) \subset M(p)\right\}$.

Definition 2.5. A Morse decomposition of $S$, denoted by $\mathscr{M}(S)=\{M(p) \mid$ $p \in P\}$, is a collection of mutually disjoint isolated invariant subsets of $S$ satisfying the following property. Given $x \in S$, then $x \in M(p)$ for some $p \in P$ or there exists $p, p^{\prime} \in P$ and a strict partial order such that $p>p^{\prime}$ and $x \in C\left(M(p), M\left(p^{\prime}\right)\right)$.

Any such ordering is called an admissible ordering for $\mathscr{M}(S)$. If we have a fixed admissible ordering in mind then we shall write $\mathscr{M}(S)=\{M(p) \mid p \in$ $(P,>)\}$. There is a minimal admissible ordering which we call the flow defined ordering and denote by $>_{\varphi}$. It is defined by

$p>_{\varphi} p^{\prime}$ if and only if there exists a sequence $p^{\prime}=p_{0}, p_{1}, \ldots, p_{n}=p$ such that $C\left(M\left(p_{i+1}\right), M\left(p_{i}\right)\right) \neq \varnothing$.

Let $I \in \mathscr{I}$, then one can define a new isolated invariant set

$$
M(I)=\left(\bigcup_{i \in I} M(i)\right) \cup\left(\bigcup_{j i \in I} C(M(j), M(i))\right) .
$$

Since $M(I)$ is an isolated invariant set $I(M(I))$ is defined.

It should be clear that given an isolated invariant set there need not be a unique Morse decomposition. A particularly simple, but useful Morse decomposition is one which consists of only two Morse sets, i.e., $\mathscr{M}(S)=\{M(p) \mid$ $p=1,2$ and $2>1\}$. In this case we refer to the Morse decomposition as an attractor-repeller pair and denote $M(1)$ by $A$, and $M(2)$ by $A^{*}$. $A$ is called an attractor of $S$ and $A^{*}$ is the dual repeller of $A$. As an example of this, consider the isolated invariant sets $S_{a}$ and $S_{b}$ of Figure 3 . Notice, that in both cases we have a Morse decomposition made up of the critical points $x$ and $y$. Since $C(x, y)=\varnothing$ and $C(y, x)=\varnothing$, we have a variety of possible partial orders, i.e., $x>y$, or $y>x$, or no relation between $x$ and $y$, all define admissible orderings on the Morse decomposition. However, for the purposes of the next section, we shall choose the ordering $x>y$ and thereby obtain an attractor repeller decomposition of $S_{a}$ and $S_{b}$ given by $\left(A, A^{*}\right)=(y, x)$.

One of the most important properties of the Conley index is that it continues under perturbation. It is this fact that allows us to obtain our bifurcation results. Let us indicate then what is meant by continuation of the index.

We begin with a collection of local semiflows $\left\{\varphi_{\lambda} \mid \lambda \in \Lambda\right.$ a metric space $\}$ defined on the metric space $X$ and parameterized by $\Lambda$. We can now define a new local semiflow $\vartheta$ on $X \times \Lambda$ by

$$
\vartheta(x, \lambda, t)=\left(\varphi_{\lambda}(x, t), \lambda\right) .
$$


$\vartheta$ is called the parameter semiflow. Let $\Pi_{X}: X \times \Lambda \rightarrow X$ and $\Pi_{\Lambda}: X \times \Lambda \rightarrow \Lambda$ denote the standard projection maps.

Lemma 2.6. Assume that $\vartheta$ is an admissible parameter semiflow on $Y \times \Lambda \subset$ $X \times \Lambda$. Let $N$ be a closed subset of $Y$, then the set $\Lambda(N)=\{\lambda \mid N \times \lambda$ is an isolating neighborhood in $X \times \lambda\}$ is open in $\Lambda$.

Definition 2.7. The space of isolated invariant sets is

$$
\begin{aligned}
& \mathscr{S}=\{S \times \lambda \mid \lambda \in \Lambda, X \times \lambda \text { is an isolated invariant set } \\
& \text { with an admissible isolating neighborhood in } \left.X \times \lambda \text { under } \varphi_{\lambda}\right\} .
\end{aligned}
$$

$\mathscr{S}$ is topologized as follows. Given a closed admissible set $N \subset X$, define $l_{N}: \Lambda(N) \rightarrow \mathscr{S}$, by $\imath_{N}(\lambda)=I(N \times \lambda)$. The open sets of $\mathscr{S}$ are generated by sets of the form $l_{N}(U)$ where $N$ is closed and admissible, and $U \subset \Lambda(N)$ is open.

A continuous map $\gamma: \Lambda \rightarrow \mathscr{S}$ is called a section of the space of isolated invariant sets if $\Pi_{\Lambda} \circ \gamma=\left.\mathrm{id}\right|_{\Lambda}$.

Lemma 2.8 [Salamon, Lemma 6.4]. (i) Let $\gamma: \Lambda \rightarrow \mathscr{S}$ be a section, then $\gamma$ is continuous if and only if

$$
S=\bigcup_{\lambda \in \Lambda} \gamma(\lambda)
$$

is an isolated invariant set in $X \times \Lambda$.

(ii) Let $\gamma: \Lambda \rightarrow \mathscr{S}$ be a section and let $\alpha: \Lambda \rightarrow \mathscr{S}$ be a continuous map such that $\alpha(\lambda)$ is an attractor in $\gamma(\lambda)$. Furthermore, let $\alpha^{*}(\lambda)$ denote the complementary repeller of $\alpha(\lambda)$ in $\gamma(\lambda)$ and let $S=\bigcup_{\lambda \in \Lambda} \gamma(\lambda)$. Then $\alpha^{*}: \Lambda \rightarrow \mathscr{S}$ is continuous and the sets

$$
A=\bigcup_{\lambda \in \Lambda} \alpha(\lambda) \text { and } A^{*}=\bigcup_{\lambda \in \Lambda} \alpha^{*}(\lambda)
$$

form an attractor repeller pair in $S$.

We are also concerned with the continuation of Morse decompositions. Let $\mathscr{M}(S)=\{M(p) \mid p \in(P,>)\}$ be an admissibly ordered Morse decomposition. We say that $\mathscr{M}(S)$ and its ordering continue over $\Lambda$ if there exists sections $\sigma, \mu_{p}: \Lambda \rightarrow \mathscr{S}$ such that $\left\{\mu_{p}(\lambda) \mid p \in(P,>)\right\}$ is a Morse decomposition of $\sigma(\lambda)$ for all $\lambda \in \Lambda$ and for some $\lambda_{0} \in \Lambda, \sigma\left(\lambda_{0}\right)=S$, and $\mu_{p}\left(\lambda_{0}\right)=M(p)$. The same proof as that for Lemma 2.8(ii) gives the following proposition.

Proposition 2.9. Let

$$
\mathbf{S}=\bigcup_{\lambda \in \Lambda} \sigma(\lambda), \quad \mathbf{M}(p)=\bigcup_{\lambda \in \Lambda} \mu_{p}(\lambda) \text { for } p \in P .
$$

Then, $\mathbf{S}$ is an isolated invariant set in $X \times \Lambda$ under $\vartheta$ and $\mathscr{M}(\mathbf{S})=\{\mathbf{M}(p) \mid$ $p \in(P,>)\}$ is a Morse decomposition of $\mathbf{S}$ if and only if $\mathscr{M}(S)$ and its ordering continue, i.e., we have sections $\sigma, \mu_{p}: \Lambda \rightarrow \mathscr{S}$ as described above.

Since $\mathscr{M}(\mathbf{S})$ is a Morse decomposition, it must have a flow defined order. To indicate that this order is admissible over all of $\Lambda$ we shall denote it by $>_{\Lambda}$. We introduced the continuation of Morse decompositions in this manner because it seems more natural in the context of bifurcation theory, i.e., one 
begins with a fixed semiflow $\varphi_{\lambda_{0}}$ and thinks of continuing it over a set $\Lambda$. However, for the purposes of presenting the theory, it is more useful to begin with $S \subset X \times \Lambda$, an isolated invariant set under $\vartheta$, the parameter flow, with a Morse decomposition $\mathscr{M}(S)=\{M(p) \mid p \in(P,>)\}$. Now by Proposition 2.9, there exist sections $\sigma, \mu_{p}: \Lambda \rightarrow \mathscr{S}$. To simplify the notation, we shall set $S_{\lambda}=\sigma(\lambda), M_{\lambda}(p)=\mu_{p}(\lambda)$, and $>_{\lambda}=>_{\varphi_{\lambda}}$.

Theorem 2.14 [Salamon, Theorem 6.7]. Let $(N, L)$ be an index pair for $S \subset$ $X \times \Lambda$. Then the canonical injection map $i(\lambda): N(\lambda) / L(\lambda) \rightarrow N / L$ induces a morphism between the connected simple systems $I\left(\sigma(\lambda), \varphi_{\lambda}\right)$ and $I(S, \vartheta)$ which is independent of the choice of the index pair $(N, L)$. Furthermore, this morphism is in fact a local isomorphism.

Notice that $F_{\lambda \lambda^{\prime}}: I\left(\sigma(\lambda), \varphi_{\lambda}\right) \rightarrow I\left(\sigma\left(\lambda^{\prime}\right), \varphi_{\lambda^{\prime}}\right)$ defined by $F_{\lambda \lambda^{\prime}}=p\left(\lambda^{\prime}\right) \circ i(\lambda)$ is an isomorphism of connected simple systems, where $p\left(\lambda^{\prime}\right)$ is the canonical projection map induced by the local isomorphism. In what follows, it is assumed that the parameter flow $\vartheta$ on $X \times \Lambda$ is fixed, thus, to simplify the notation, let $I_{\lambda}(\sigma(\lambda))=I\left(\sigma(\lambda), \varphi_{\lambda}\right)$.

\section{TRANSITIONS OF CONNECTED SIMPLE SYSTEMS}

As has been stated before, we intend to use connected simple systems in order to detect global bifurcations. The obvious first question and the one we intend to answer in this section is: how does one measure the difference between connected simple systems?

Let $X \times \Lambda$ be a parameterized family of semiflows as discussed in $\S 2$, with $\Lambda$ a path connected space. Assume that $\Pi_{1}(\Lambda)$ acts trivially on $\mathscr{S}$ (i.e., given $\sigma$ a section in $\mathscr{S}$, the index isomorphism $F_{\lambda \lambda^{\prime}}$, from $I_{\lambda}(\sigma(\lambda))$ to $I_{\lambda^{\prime}}\left(\sigma\left(\lambda^{\prime}\right)\right)$ is independent of the path chosen). Let $S \subset X \times \Lambda$ be an isolated invariant set with Morse decomposition $\mathscr{M}(S)=\{M(p) \mid p \in(P,>)\}$. Define

$$
\Lambda^{\prime}=\left\{\lambda \in \Lambda \mid S_{\lambda}=\bigcup_{p \in P} M_{\lambda}(p)\right\} \subset \Lambda .
$$

Notice that $\Lambda^{\prime}$ is the set of parameter values for which no connecting orbits between Morse sets occur. For every $J \in \mathscr{I}(>)$ and every $\lambda \in \Lambda^{\prime}$ the connected simple system $I_{\lambda}\left(M_{\lambda}(J)\right)=I_{\lambda}\left(\bigcup_{p \in J} M_{\lambda}(p)\right)$ can be partitioned into two subcategories

$D_{\lambda}(J)=\left\{[N / L] \in I_{\lambda}\left(M_{\lambda}(J)\right) \mid\right.$ every component of $N$ intersects at most one Morse set $\left.M_{\lambda}(p)\right\}$ and

$$
I_{\lambda}(J)=I_{\lambda}\left(M_{\lambda}(J)\right) \backslash D_{\lambda}(J) \text {. }
$$

Notice that the morphisms in $I_{\lambda}\left(M_{\lambda}(J)\right)$ from $D_{\lambda}(J)$ to $I_{\lambda}(J)$ define an isomorphism $\Phi_{\lambda}(J)$ of connected simple systems.

As $M(J)$ continues across $\Lambda$, for any $\lambda, \mu \in \Lambda^{\prime}$, there exists an index isomorphism

$$
F_{\lambda \mu}(J): I_{\lambda}\left(M_{\lambda}(J)\right) \rightarrow I_{\mu}\left(M_{\mu}(J)\right) .
$$

Similarly, continuation of $\bigcup_{p \in J} M(p)$ defines an index isomorphism

$$
E_{\lambda \mu}(J): I_{\lambda}\left(\bigcup_{p \in J} M_{\lambda}(p)\right) \rightarrow I_{\mu}\left(\bigcup_{p \in J} M_{\mu}(p)\right)
$$


The difference between the two is that for $\nu \notin \Lambda^{\prime}, M_{\nu}(J)$ will contain connecting orbits, and $F_{\lambda \mu}(J)$ will (in general) reflect the changes in the connected simple systems caused by those connections. On the other hand, for all $\nu \in \Lambda$, $\bigcup_{p \in J} M_{\nu}(p)$ explicitly has no connecting orbits, and therefore, $E_{\lambda} \mu(J)$ cannot detect any changes when passing through $\Lambda / \Lambda^{\prime}$. More succinctly, it is possible that $E_{\lambda \mu}(J) \neq F_{\lambda \mu}(J)$.

When restricted to the appropriate subcategories, $F_{\lambda \mu}(J)$ and $E_{\lambda \mu}(J)$ define index isomorphisms

$$
F_{\lambda \mu}(J): I_{\lambda}(J) \rightarrow I_{\mu}(J) \text { and } E_{\lambda \mu}(J): D_{\lambda}(J) \rightarrow D_{\mu}(J)
$$

Thus, the difference in connected simple systems at $\lambda$ and $\mu$ may be measured by the failure of the following diagram to commute.

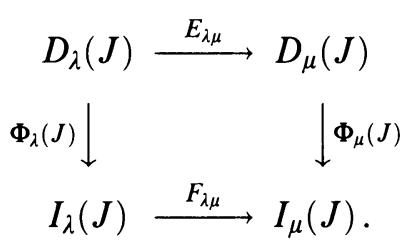

The connected simple systems $I_{\lambda}\left(M_{\lambda}(J)\right)$ and $I_{\mu}\left(M_{\mu}(J)\right)$ will be called equivalent (simply denoted $I_{\lambda}\left(M_{\lambda}(J)\right) \cong I_{\mu}\left(M_{\mu}(J)\right)$ ) if the diagram (3.1) commutes. Define

$$
\left.T_{\lambda \mu}(J)=\Phi_{\mu}^{-1}(J) \circ F_{\lambda \mu}(J) \circ \Phi_{\lambda}(J)\right)
$$

Keeping in mind the assumption that $\Pi_{1}(\Lambda)$ acts trivially on $\mathscr{S}$, the discussion up to this point can be summarized in the following proposition.

Proposition 3.1. (i) $E_{\lambda \rho}(J)=E_{\mu \rho}(J) \circ E_{\lambda \mu}(J)$.

(ii) $T_{\lambda \rho}(J)=T_{\mu \rho}(J) \circ T_{\lambda \mu}(J)$.

(iii) $I_{\lambda}\left(M_{\lambda}(J)\right) \cong I_{\mu}\left(M_{\mu}(J)\right)$ if and only if $E_{\lambda \mu}(J)=T_{\lambda \mu}(J)$.

(iv) If $\lambda, \mu \in \Lambda$ lie in the same path component of $\Lambda^{\prime}$, then $I_{\lambda}\left(M_{\lambda}(J)\right) \cong$ $I_{\mu}\left(M_{\mu}(J)\right)$ for all $J \in \mathscr{I}(P,>)$.

Returning to Figure 3 , let us consider why $I_{a}\left(S_{a}\right) ¥ I_{b}\left(S_{b}\right)$. The first step is to choose generators $\alpha_{\lambda}$ and $\beta_{\lambda}$ of $D_{\lambda}=D_{\lambda}(P)$ and generators $\gamma_{\lambda}$ and $\delta_{\lambda}$ of $I_{\lambda}=I_{\lambda}(P)$ where $\lambda=a, b$. Figure 4 indicates such choices. Now consider the diagram obtained from (3.1).

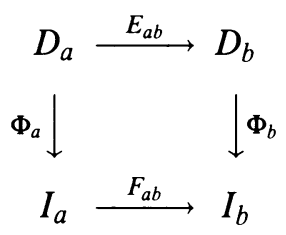

and notice that

$$
\begin{array}{ll}
E_{a b}\left(\alpha_{a}\right)=\alpha_{b}, & E_{a b}\left(\beta_{a}\right)=\beta_{b}, \\
F_{a b}\left(\gamma_{a}\right)=\gamma_{b}, & F_{a b}\left(\delta_{a}\right)=\delta_{b}, \\
\Phi_{a}\left(\alpha_{a}\right)=\gamma_{a}, & \Phi_{a}\left(\beta_{a}\right)=\beta_{a}, \\
\Phi_{b}\left(\alpha_{a}\right)=\delta_{p} * \gamma_{b}, & \Phi_{b}\left(\beta_{b}\right)=\delta_{b}
\end{array}
$$



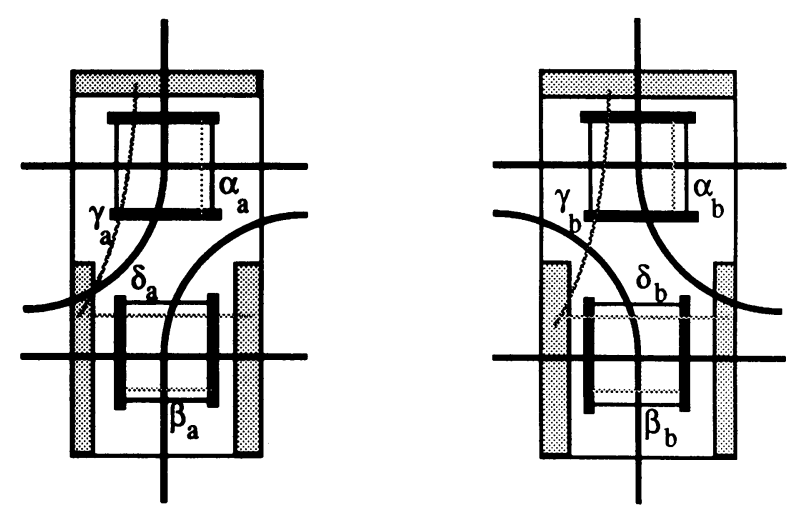

FIGURE 4

Now one sees that

$$
F_{a b} \Phi_{a}\left(\alpha_{a}\right)=F_{a b}\left(\gamma_{a}\right)=\gamma_{b} \neq \delta_{b} * \gamma_{b}=\Phi_{b}\left(\alpha_{b}\right)=\Phi_{b} E_{a b}\left(\alpha_{b}\right) .
$$

Therefore $I_{a}\left(S_{a}\right) \not I_{b}\left(S_{b}\right)$.

A number of simple examples show that the converse to 3.1 (iv) is false. Of interest to us is the fact that $E_{\lambda \mu}(J) \neq T_{\lambda \mu}(J)$ implies the existence of a connecting orbit at some parameter value between $\lambda$ and $\mu$. In fact, the set of parameter values for which a connection occurs must separate $\lambda$ and $\mu$ in $L$.

As the example above illustrates, diagram (3.1) does not commute in general, but there are pieces which do commute for all $\lambda, \mu \in \Lambda^{\prime}$. If $(J, K) \in$ $\mathscr{I}_{2}(P,>)$, there are for every $\lambda \in \Lambda$ natural projection and inclusion morphisms $i_{\lambda}(J, J K): D_{\lambda}(J) \rightarrow D_{\lambda}(J K), p_{\lambda}(J K, K): D_{\lambda}(J K) \rightarrow D_{\lambda}(K)$, etc., with a commutative diagram

$$
\begin{array}{ccc}
D_{\lambda}(J) \stackrel{i_{\lambda}(J, J K)}{\longrightarrow} D_{\lambda}(J K) \stackrel{p_{\lambda}(J K, K)}{\longrightarrow} D_{\lambda}(K) \\
\Phi_{\lambda}(J) \downarrow & \Phi_{\lambda}(J K) \downarrow & \\
I_{\lambda}(J) \stackrel{i_{\lambda}(J, J K)}{\longrightarrow} I_{\lambda}(J K) \stackrel{\Phi_{\lambda}(J K, K)}{\longrightarrow} & I_{\lambda}(K)
\end{array}
$$

Further, there are commutative diagrams

$$
\begin{array}{ccc}
D_{\lambda}(J) \stackrel{i_{\lambda}(J, J K)}{\longrightarrow} D_{\lambda}(J K) \stackrel{p_{\lambda}(J K, K)}{\longrightarrow} & D_{\lambda}(K) \\
E_{\lambda \mu}(J) \downarrow & E_{\lambda \mu}(J K) \downarrow & \\
D_{\mu}(J) \stackrel{i_{\lambda \mu}(K)}{ } \stackrel{\downarrow}{\longrightarrow} D_{\mu}(J K) \stackrel{p_{\mu}(J K, K)}{\longrightarrow} D_{\mu}(K)
\end{array}
$$

and

$$
\begin{array}{ccc}
I_{\lambda}(J) \stackrel{i_{\lambda}(J, J K)}{\longrightarrow} I_{\lambda}(J K) \stackrel{p_{\lambda}(J K, K)}{\longrightarrow} & I_{\lambda}(K) \\
F_{\lambda \mu}(J) \downarrow & F_{\lambda \mu}(J K) \downarrow & \\
I_{\mu}(J) \stackrel{i_{\lambda}(J, J K)}{\longrightarrow} I_{\mu}(J K) \stackrel{p_{\mu}(J K, K)}{\longrightarrow} I_{\mu}(K)
\end{array}
$$


for all $\lambda, \mu \in \Lambda^{\prime}$. Composing these, there is also a commutative diagram

$$
\begin{array}{ccc}
D_{\lambda}(J) \stackrel{i_{\lambda}(J, J K)}{\longrightarrow} D_{\lambda}(J K) \stackrel{p_{\lambda}(J K, K)}{\longrightarrow} & D_{\lambda}(K) \\
T_{\lambda \mu(J)} \downarrow & T_{\lambda \mu}(J K) \downarrow & \\
D_{\mu}(J) \stackrel{i_{\mu}(J, J K)}{\longrightarrow} D_{\mu}(J K) \stackrel{p_{\mu}(J K, K)}{\longrightarrow} & D_{\mu}(K) .
\end{array}
$$

From these we obtain relations between the maps which imply partial equality of the maps $E$ and $T$.

Proposition 3.2. If $J \subset K$ are intervals in $(P,>)$ and $p, q \in J$, then

$$
\begin{gathered}
p_{\mu}(J, q) \circ E_{\lambda \mu}(J) \circ i_{\lambda}(p, J)=p_{\mu}(K, q) \circ E_{\lambda \mu}(K) \circ i_{\lambda}(p, K), \\
p_{\mu}(J, q) \circ T_{\lambda \mu}(J) \circ i_{\lambda}(p, J)=p_{\mu}(K, q) \circ T_{\lambda \mu}(K) \circ i_{\lambda}(p, K) .
\end{gathered}
$$

That is, the $p, q$ entries of $E_{\lambda \mu}$ and $T_{\lambda \mu}$ are independent of the interval which contains $p$ and $q$.

Proof. The inclusions and projections are related by

$$
i_{\lambda}(J, K) \circ i_{\lambda}(p, J)=i_{\lambda}(p, K)
$$

and

$$
p_{\mu}(J, q) \circ p_{\mu}(K, J)=p_{\mu}(K, q) .
$$

Thus it suffices to show that

$$
E_{\lambda \mu}(J)=p_{\mu}(K, J) \circ E_{\lambda \mu}(K) \circ i_{\lambda}(J, K)
$$

and

$$
T_{\lambda \mu}(J)=p_{\mu}(K, J) \circ T_{\lambda \mu}(K) \circ i_{\lambda}(J, K) .
$$

Furthermore, there exists $Q \in \mathscr{I}(P,>)$ for which $J \subset Q \subset K$, and such that $J$ is an attractor in $Q$ and $Q$ is a repeller in $K$. Thus it is sufficient to assume that $J$ is either an attractor or a repeller in $K$.

If $J$ is an attractor in $K$, then the diagram (3.5) implies that

$$
i_{\lambda}(J, K) \circ E_{\lambda \mu}(J)=E_{\lambda \mu}(K) \circ i_{\lambda}(J, K) .
$$

For $\mu \in \Lambda^{\prime}, M(J)=\bigcup_{p \in J} M_{\mu}(p)$, so $i_{\mu}$ and $p_{\mu}$ are just inclusion and projection of factors. In particular, $p_{\mu}(K, J) \circ i_{\mu}(J, K)=$ id and

$$
E_{\lambda \mu}(J)=p_{\mu}(K, J) \circ i_{\mu}(J, K) \circ E_{\lambda \mu}(J)=p_{\mu}(K, J) \circ E_{\lambda \mu}(K) \circ i_{\lambda}(J, K) .
$$

Similarly, if $J$ is a repeller in $K$, we have, again via diagram (3.5), that

$$
p_{\mu}(K, J) \circ E_{\lambda \mu}(K)=E_{\lambda \mu}(J) \circ p_{\lambda}(K, J)
$$

and so

$$
p_{\mu}(K, J) \circ E_{\lambda \mu}(K) \circ i_{\lambda}(J, K)=E_{\lambda \mu}(J) \circ p_{\lambda}(K, J) \circ i_{\lambda}(J, K)=E_{\lambda \mu}(J) .
$$

The argument for (3.9) is similar.

Proposition 3.3. If $p$ is an attractor in $J$, then

$$
E_{\lambda \mu}(J) \circ i_{\lambda}(p, J)=T_{\lambda \mu}(J) \circ i_{\lambda}(p, J) .
$$


Proof. Since $p$ is an attractor,

$$
i_{\mu}(p, J) \circ E_{\lambda \mu}(p)=E_{\lambda \mu}(J) \circ i_{\lambda}(p, J)
$$

and

$$
\begin{aligned}
T_{\lambda \mu}(J) \circ i_{\lambda}(p, J) & =i_{\mu}(p, J) \circ T_{\lambda \mu}(p) \\
& =i_{\mu}(p, J) \circ \Phi_{\mu}^{-1}(p) \circ F_{\lambda \mu}(p) \circ \Phi_{\lambda}(p) .
\end{aligned}
$$

However, $M(p)$ continues over all of $\Lambda$, thus $E_{\lambda \mu}(p)$ and $F_{\lambda \mu}(p)$ define the same morphism of connected simple systems, i.e.,

$$
E_{\lambda \mu}(p)=\Phi_{\mu}^{-1}(p) \circ F_{\lambda \mu}(p) \circ \Phi_{\lambda}(p) \text {. }
$$

Let $K \subset \Lambda$ be a path connected subset. Define $S_{K}=S \cap(X \times K)$ and $\vartheta_{K}=$ $\left.\vartheta\right|_{X \times K}$. Then clearly the Morse decomposition $\mathscr{M}(S)=\{M(p) \mid p \in(P,>)\}$ restricts to a Morse decomposition $\mathscr{M}\left(S_{K}\right)$. Notice that the flow defined ordering on $\mathscr{M}\left(S_{K}\right)$ need not be the same as the flow defined ordering on $\mathscr{M}(S)$. Let $>_{K}$ denote the flow defined order on $\mathscr{M}\left(S_{K}\right)$.

Proposition 3.4. Let $p, q \in P$, let $\lambda, \mu \in \Lambda^{\prime}$, and let $K \subset \Lambda$. If $p$ and $q$ are unrelated in the flow defined partial order $>_{K}$, then

$$
p_{\mu}(P, q) \circ T_{\lambda \mu}(P) \circ i_{\lambda}(p, P)=p_{\mu}(P, q) \circ E_{\lambda \mu}(P) \circ i_{\lambda}(p, P) .
$$

Proof. $K$ defines a continuation from $\lambda$ to $\mu$. If $p$ and $q$ are unrelated, then $\{p, q\}$ is an interval in $P$. Thus,

$$
p_{\mu}(\{p, q\}, q) \circ E_{\lambda \mu}(\{p, q\}) \circ i_{\lambda}(p,\{p, q\})=p_{\mu}(P, q) \circ E_{\lambda \mu}(P) \circ i_{\lambda}(p, P)
$$

and

$$
p_{\mu}(\{p, q\}, q) \circ T_{\lambda \mu}(\{p, q\}) \circ i_{\lambda}(p,\{p, q\})=p_{\mu}(P, q) \circ T_{\lambda \mu}(P) \circ i_{\lambda}(p, P) .
$$

Since there are no connecting orbits in $\{p, q\}$ over $K$,

$$
p_{\mu}(\{p, q\}, q) \circ E_{\lambda \mu}(\{p, q\}) \circ i_{\lambda}(p,\{p, q\})=p_{\mu}(P, q) \circ T_{\lambda \mu}(P) \circ i_{\lambda}(p, P) \text {. }
$$

This proposition tells us that $E_{\lambda \mu}(P)$ and $T_{\lambda \mu}(P)$ agree on "lower-triangular entries," i.e., those $p, q$ entries with $p \ngtr q$.

As has been remarked several times in the introduction and as is, perhaps, clear at this point, working on this level of homotopy theory can be fairly difficult. Therefore, we want to conclude this section with remarks on how this theory presents itself in the context of a homology (or cohomology) theory. The homology functor $\mathrm{CH}_{*}$ (equivalently the cohomology function $\mathrm{CH}^{*}$ ) can be applied to all these connected simple systems and hence, morphisms between connected simple systems induce homomorphisms between the induced homology groups. We will abuse notation and use the same symbol for an index morphism and its induced homology map. Notice that for all $\lambda \in \Lambda^{\prime}$ and $J \in \mathscr{I}\left(P,>_{\lambda}\right)$, there is a natural isomorphism between

$$
\bigoplus_{p \in J} C H_{*}\left(M_{\lambda}(p), \varphi^{\lambda}\right) \text { and } C H_{*}\left(\bigcup_{p \in J} M_{\lambda}(p), \varphi^{\lambda}\right) \text {. }
$$

We will identify the two groups via this isomorphism and write

$$
C_{*} \Delta_{\lambda}(J)=\bigoplus_{p \in J} C H_{*}\left(M_{\lambda}(p), \varphi^{\lambda}\right) \text {. }
$$


The reason for this notation will become clear in $\S 5$ when connection matrices are introduced.

$\mathrm{CH}_{*}$ takes index morphisms to homology homomorphism and index isomorphisms to homology isomorphisms. Suppose that on $\Lambda, \mathrm{CH}_{*}$ is faithful, i.e., if $f, g: I\left(S_{\lambda}, \varphi_{\lambda}\right) \rightarrow I\left(S_{\mu}, \varphi_{\mu}\right)$ are morphisms of connected simple systems, then $f=g$ if and only if $f_{*}=g_{*}: C H_{*}\left(S_{\lambda}, \varphi_{\lambda}\right) \rightarrow C H_{*}\left(S_{\mu}, \varphi_{\mu}\right)$. This will be the case, for instance, when all Morse sets have the index of an $n$-sphere. Then, the connected simple systems $I_{\lambda}\left(S_{\lambda}(J)\right)$ and $I_{\mu}\left(S_{\mu}(J)\right)$ are equivalent if and only if

$$
\begin{array}{ccc}
C_{*} \Delta(J, \lambda) & \stackrel{F_{\lambda \mu}(J)}{\longrightarrow} & C_{*} \Delta(J, \mu) \\
\downarrow \Phi_{\lambda}(J) & & \Phi_{\mu}(J) \\
C H_{*}\left(S_{\lambda}(J), \varphi_{\lambda}\right) \stackrel{F_{\lambda \mu}(J)}{\longrightarrow} C H_{*}\left(S_{\mu}(J), \varphi_{\mu}\right)
\end{array}
$$

commutes.

Stated more simply, one can study the differences in the connected simple systems by comparing the isomorphisms $E_{\lambda \mu}$ and $T_{\lambda \mu}$ from $C_{*} \Delta_{\lambda}(J)$ to $C_{*} \Delta_{\mu}(J)$. Towards this end, fix a $\lambda_{0} \in \Lambda^{\prime}$ and a choice of generators for $C_{*} \Delta_{0}(J)=C_{*} \Delta_{\lambda_{0}}(J)$. Now use $E_{\lambda_{0} \lambda}$ to fix generators for all other $\lambda \in \Lambda^{\prime}$. Since $E_{\lambda \mu} \circ E_{\lambda_{0} \mu}=E_{\lambda_{0} \mu}, E_{\lambda \mu}$ is represented by the identity matrix in this system of coordinates. Using $E_{\lambda \mu}(J)$ in this way to identify generators, $T_{\lambda \mu}(J)$ can be represented as a matrix mapping from $C_{*} \Delta_{\lambda}(J)$ to $C_{*} \Delta_{\mu}(J)$, where the $p, q$ entry of the matrix

$$
\left[T_{\lambda \mu}(J)\right]_{p q}: C H_{*}\left(M_{\lambda}(q), \varphi^{\lambda}\right) \rightarrow C H_{*}\left(M_{\mu}(p), \varphi^{\mu}\right)
$$

is given by

$$
\left[T_{\lambda \mu}(J)\right]_{p q}=p_{\mu}(J, p) \circ T_{\lambda \mu}(J) \circ i_{\lambda}(q, J) .
$$

Summarizing, we have the following proposition.

Proposition 3.5. Let $J \subset K$ be intervals in $P$, with $p, q \in K$, then: $J$.

(i) $T_{\lambda \mu}(J)$ is a submatrix of $T_{\lambda \mu}(K)$, i.e., $\left[T_{\lambda \mu}(J)\right]_{p q}=\left[T_{\lambda \mu}(K)\right]_{p q}$ for $p, q \in$ id.

(ii) $T_{\lambda \mu}(J)$ is upper triangular, i.e., $\left[T_{\lambda \mu}(J)\right]_{p q}=0$ if $q \ngtr p$ and $\left[T_{\lambda \mu}(J)\right]_{p p}=$

(iii) $T_{\lambda \mu}(J)$ detects changes in the connected simple system, i.e., $I\left(M_{\lambda}(J), \varphi^{\lambda}\right)$ $\cong I\left(M_{\mu}(J), \varphi^{\mu}\right)$ if and only if $T_{\lambda \mu}(J)=\mathrm{id}$.

\section{THE BIFURCATION RESULT}

In this section we state and prove the desired bifurcation results. We begin by stating three assumptions and finish with a comparison between our results and those of Chow, Deng and Terman.

Using the notation of the previous sections we shall assume

A1. $S \subset X \times \Lambda$ is an isolated invariant set under $\vartheta$. Furthermore, $\mathscr{M}(S)=$ $\{M(p) \mid p=1,2$, or 3 with $3>2>1\}$ is a Morse decomposition of $S$.

A2. $I\left(M_{\lambda}(p), \varphi_{\lambda}\right) \sim \Sigma^{n}, n \geq 1$ for $p=1,2,3$ and for all $\lambda$.

A3. There exist parameter values $\lambda_{0}, \lambda_{1}, \lambda_{2}, \lambda_{3} \in \Lambda^{\prime}$ for which $I_{0}(1,2)$, $I_{1}(1,2) \not I_{2}(1,2), I_{3}(1,2)$ and $I_{0}(2,3), I_{2}(2,3) \not I_{1}(2,3), I_{3}(2,3)$ (where $\left.I_{i}(J)=I\left(M_{\lambda_{i}}(J), \varphi_{\lambda_{i}}\right)\right)$.

Let $C_{i j}=\left\{\lambda \in \Lambda \mid\right.$ there exists a connecting orbit from $M_{\lambda}(i)$ to $\left.M_{\lambda}(j)\right\}$. 
Theorem 4.1. Assume A1-A3. Then $C_{21}$ separates $\left\{\lambda_{0}, \lambda_{1}\right\}$ from $\left\{\lambda_{2}, \lambda_{3}\right\}$ and $C_{32}$ separates $\left\{\lambda_{0}, \lambda_{2}\right\}$ from $\left\{\lambda_{1}, \lambda_{3}\right\}$.

Proof. This follows immediately from Proposition 3.1(iv) and A3.

Theorem 4.2. Assume $\mathrm{A} 1-\mathrm{A} 3$ and further assume that $I_{0}(1,2) \cong I_{1}(1,2)$, $I_{2}(1,2) \cong I_{3}(1,2), I_{0}(2,3) \cong I_{2}(2,3)$, and $I_{1}(2,3) \cong I_{3}(2,3)$. Then one of the following occurs:

(i) $C_{21} \cup C_{31}$ separates either $\lambda_{0}$ from $\lambda_{1}$ or $\lambda_{2}$ from $\lambda_{3}$,

(ii) $C_{32} \cup C_{31}$ separates either $\lambda_{0}$ from $\lambda_{2}$ or $\lambda_{1}$ from $\lambda_{3}$.

Corollary 4.3. Assume A1-A 3 and further assume that $C_{21}$ does not separate $\lambda_{0}$ from $\lambda_{1}$ nor $\lambda_{2}$ from $\lambda_{3}$, and that $C_{32}$ does not separate $\lambda_{0}$ from $\lambda_{1}$ nor $\lambda_{2}$ from $\lambda_{3}$. Then $C_{31} \neq \varnothing$, and one of the following occurs

(i) $C_{21} \cup C_{31}$ separates either $\lambda_{0}$ from $\lambda_{1}$ or $\lambda_{2}$ from $\lambda_{3}$,

(ii) $C_{32} \cup C_{31}$ separates either $\lambda_{0}$ from $\lambda_{2}$ or $\lambda_{1}$ from $\lambda_{3}$.

Proof. If $C_{21}$ does not separate either $\left\{\lambda_{0}, \lambda_{1}\right\}$ or $\left\{\lambda_{2}, \lambda_{3}\right\}$, then $I_{0}(12) \cong$ $I_{1}(12)$ and $I_{2}(12) \cong I_{3}(12)$. Similarly, if $C_{32}$ does not separate either $\left\{\lambda_{0}, \lambda_{2}\right\}$ or $\left\{\lambda_{1}, \lambda_{3}\right\}$, then $I_{0}(23)=I_{2}(23)$ and $I_{1}(23)=I_{3}(23)$.

A typical case of Corollary 4.3 is shown in Figure 5.

Remark 4.4. Given A2 and any interval $J, D_{\lambda}(J)$ has the homotopy type of a wedge of $|J| n$-spheres. If $n=1$, then the classes of homotopy equivalences of such a wedge is bijective with $\operatorname{Aut}\left(F_{|J|}\right)$, the automorphisms of the free group on $|J|$ generators. Similarly, for $n>1$, obstruction theory gives that $\left[D_{\lambda}(J), D_{\mu}(J)\right] \approx \operatorname{Hom}\left(\mathscr{Z}^{|J|}, \mathscr{Z}^{|J|}\right)$, and that the classes of homotopy equivalences are bijective with $\operatorname{Aut}\left(\mathscr{Z}^{|J|}\right)$. Furthermore, the Hurewicz homomorphism is an isomorphism for $n>1$, so $C H_{*}$ is faithful on index morphisms. Each $T_{\lambda \mu}(J)$ is then represented by an upper triangular matrix with 1's on the diagonal. However, it is not known whether all integer upper triangular matrices can arise as the $T$ 's.

Proof of Theorem 4.2. If not, then there exist paths $\omega_{i j}:[0,1] \rightarrow \Lambda$ from $\lambda_{i}$ to $\lambda_{j}$ with $\omega_{01}$ and $\omega_{23}$ intersecting $C_{32}$ but not $C_{21} ; \omega_{13}$ and $\omega_{02}$ intersecting $C_{21}$ but not $C_{32}$; and no $\omega_{i j}$ intersecting $C_{31} \cup\left(C_{32} \cap C_{21}\right)$.

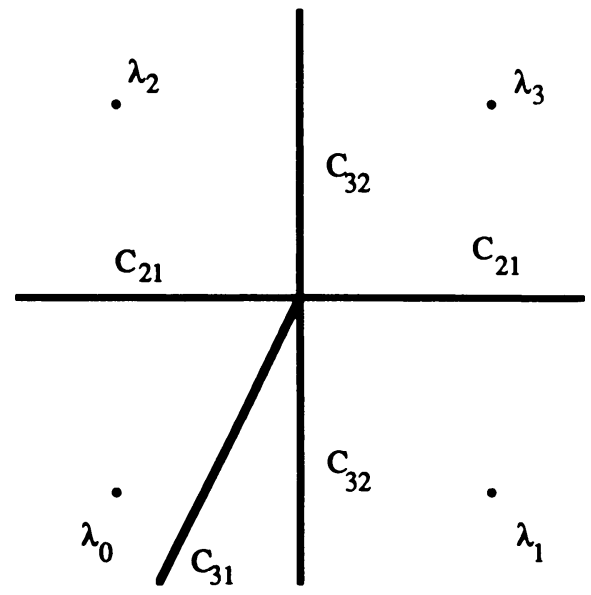

FigURE 5 
For $n>1$, these paths may be used to define the matrices $T_{i j}$ on the homology index. From Proposition 3.5, these matrices have the form

$$
\begin{array}{lll}
T_{01}=\left(\begin{array}{lll}
1 & 0 & 0 \\
0 & 1 & a \\
0 & 0 & 1
\end{array}\right), & T_{13}=\left(\begin{array}{lll}
1 & b & 0 \\
0 & 1 & 0 \\
0 & 0 & 1
\end{array}\right), \\
T_{02}=\left(\begin{array}{lll}
1 & c & 0 \\
0 & 1 & 0 \\
0 & 0 & 1
\end{array}\right), & T_{23}=\left(\begin{array}{lll}
1 & 0 & 0 \\
0 & 1 & d \\
0 & 0 & 1
\end{array}\right),
\end{array}
$$

with $a, b, c, d \neq 0$. Multiplication then yields $T_{13} T_{01} \neq T_{23} T_{02}$. Of course this contradicts the fact that $T_{13} T_{01}=T_{03}=T_{23} T_{02}$.

Note that the computation of the 1,2 and 2, 3 entries shows that $a=d$ and $b=c$, so that the matrices must in fact have the form

$$
\begin{aligned}
T_{01}=\left(\begin{array}{ccc}
1 & 0 & t_{01} \\
0 & 1 & a \\
0 & 0 & 1
\end{array}\right), & T_{13}=\left(\begin{array}{ccc}
1 & b & t_{13} \\
0 & 1 & 0 \\
0 & 0 & 1
\end{array}\right), \\
T_{02}=\left(\begin{array}{ccc}
1 & b & t_{02} \\
0 & 1 & 0 \\
0 & 0 & 1
\end{array}\right), & T_{23}=\left(\begin{array}{ccc}
1 & 0 & t_{23} \\
0 & 1 & a \\
0 & 0 & 1
\end{array}\right) .
\end{aligned}
$$

Furthermore, $C_{21} \cup C_{31}$ or $C_{32} \cup C_{31}$ separates $\lambda_{i}$ and $\lambda_{j}$ if and only if $t_{i j} \neq 0$. For $n=1$, the argument is slightly different. In this case, connected simple systems are not represented faithfully by homology automorphisms (since the Hurewicz map is not injective), but are represented faithfully by automorphisms of the fundamental group $\pi_{1}\left(M_{\lambda}, S_{\lambda}\right) \cong F_{3}$, the free group on three generators. If $\alpha_{\lambda i}$ is the generator of $\pi_{1}\left(M_{\lambda}, S_{\lambda}\right) \approx \mathscr{Z}$, then $\left\{\alpha_{\lambda 1}, \alpha_{\lambda 2}, \alpha_{\lambda 1}\right\}$ are the generators of $\pi_{l}\left(M_{\lambda}, S_{\lambda}\right)$. Let $i_{\lambda i}$ and $p_{\lambda i}$ denote the inclusion of $\left\langle\alpha_{\lambda i}\right\rangle$ into $\left\langle\alpha_{\lambda 1}, \alpha_{\lambda 2}, \alpha_{\lambda 1}\right\rangle$ and projection of $\left\langle\alpha_{\lambda 1}, \alpha_{\lambda 2}, \alpha_{\lambda_{1}}\right\rangle$ onto $\left\langle\alpha_{\lambda i}\right\rangle$.

Now consider the maps $T_{i j}$ on the fundamental group. Properties of these maps (from Propositions 3.3 and 3.4) are

$$
T_{01}\left(\alpha_{01}\right)=\alpha_{11}, T_{01}\left(\alpha_{02}\right)=\alpha_{12}, p_{11} T_{01}\left(\alpha_{03}\right)=1, p_{12} T_{01}\left(\alpha_{03}\right)=\alpha_{12}^{a} \neq 1,
$$
$p_{13} T_{01}\left(\alpha_{03}\right)=\alpha_{13}$,

$T_{13}\left(\alpha_{11}\right)=\alpha_{31}, T_{13}\left(\alpha_{13}\right)=\alpha_{33}, p_{33} T_{13}\left(\alpha_{12}\right)=1, p_{31} T_{13}\left(\alpha_{12}\right)=\alpha_{31}^{b} \neq 1$, $p_{32} T_{13}\left(\alpha_{12}\right)=\alpha_{32}$,

$T_{02}\left(\alpha_{01}\right)=\alpha_{21}, T_{02}\left(\alpha_{03}\right)=\alpha_{23}, p_{23} T_{02}\left(\alpha_{02}\right)=1, p_{21} T_{02}\left(\alpha_{02}\right)=\alpha_{21}^{c} \neq 1$, $p_{22} T_{02}\left(\alpha_{02}\right)=\alpha_{22}$,

$T_{23}\left(\alpha_{21}\right)=\alpha_{31}, T_{23}\left(\alpha_{22}\right)=\alpha_{32}, p_{31} T_{23}\left(\alpha_{23}\right)=1, p_{32} T_{23}\left(\alpha_{23}\right)=\alpha_{32}^{d} \neq 1$, $p_{33} T_{23}\left(\alpha_{23}\right)=\alpha_{33}$.

Once again, we wish to compare $T_{13} T_{01}$ with $T_{23} T_{02}$. Using the above relations one concludes that

$$
p_{13} T_{13} T_{01}\left(\alpha_{03}\right)=p_{13} T_{13}\left(\alpha_{13} \alpha_{12}^{a}\right)=p_{13}\left(\alpha_{33} \alpha_{32}^{a} \alpha_{31}^{a b}\right)=\alpha_{31}^{a b} \neq 1 .
$$

Also,

$$
p_{31} T_{23} T_{02}\left(\alpha_{03}\right)=p_{31} T_{23}\left(\alpha_{23}\right)=1 .
$$

Exactly the same contradiction as in the case $n>1$.

Let us now compare these results with those of Theorems 1.1 and 1.2. Notice that assumptions (H1), (H2) and (H3) imply A1. The allowance of $a=c$ in 

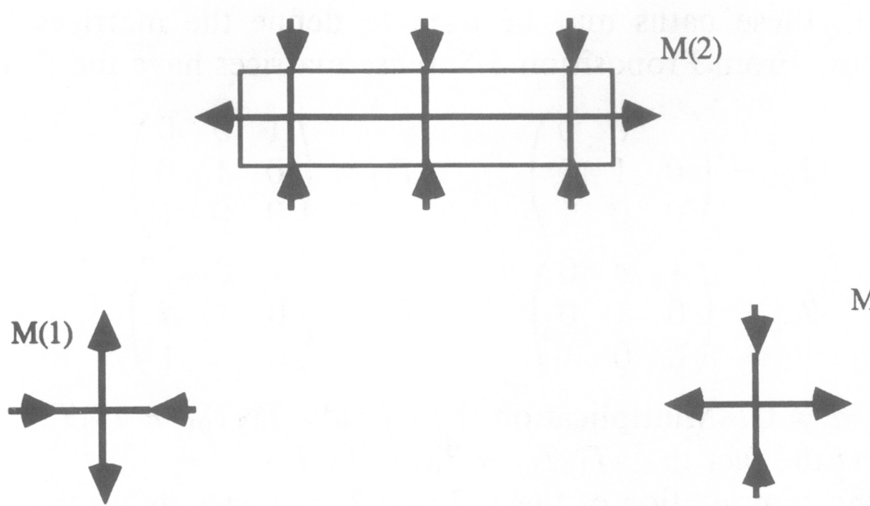

FIGURE 6

$(\mathrm{H} 2)$ is not really weaker than our A1 since the proof of [CDT] proceeds by separating the single point into two; the same can be done in our construction. It is obvious that (H4) implies A2. The relationship between (H5), (H6), and A3, might be less clear. However, one can use the transversality to show that a change in the connected simple systems occur and hence one has the existence of the $\left\{\lambda_{i}\right\} \subset \Lambda^{\prime}$.

With regards to the results, Theorem 4.2 implies Theorem 1.1 in the following sense: if $\Lambda$ is a square in $\mathbf{R}^{2}$, the $C_{21}$ and $C_{32}$ contain arcs connecting opposite sides of the square. Given the additional assumption of Theorem 1.2 (i.e., that $C_{a b}$ and $C_{b c}$ meet transversely at 0 ), the set $\operatorname{cl}_{\Lambda}\left(C_{31}\right) \cap C_{21} \cap C_{32} \neq \varnothing$, as is easilay seen by choosing the $\lambda_{i}$ 's sufficiently close to the origin.

Figures 5 and 6 indicate in part why the conclusions of Theorems 4.1, 4.2 and Corollary 4.3 are stated in such a weak form. Figure 6 indicates the Morse sets $M(3), M(2)$, and $M(1)$, where $M(2)$ is the invariant set inside the box.

Figure 7 indicates what the corresponding bifurcation diagram might look like.

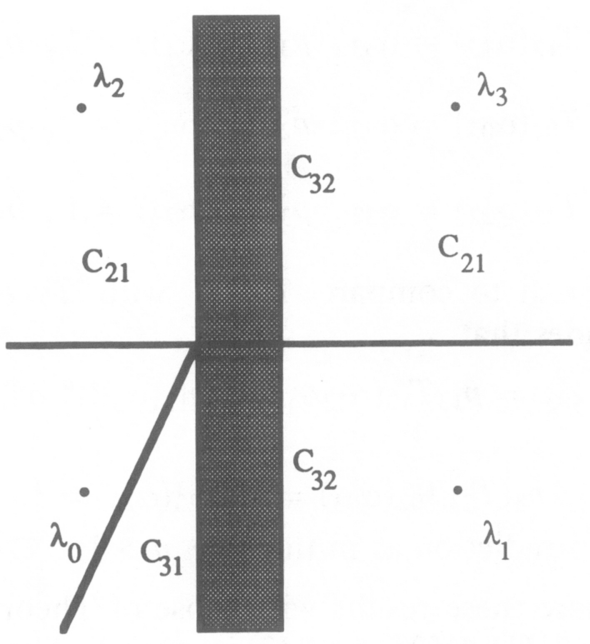

FIGURE 7 
The point of this example is to remind the reader that the individual Morse sets can be fairly complicated under our hypothesis (to make $M(2)$ more complicated, insert your favorite invariant set inside the box; the only restriction is that the flow on the boundary of the box should agree with that of Figure 5).

\section{CONNECTION MATRICES AND TRANSITION SYSTEMS}

This section begins with a review of the algebraic theory behind the connection matrix [F2, FM, Mo]. Most of the results concerning this are due to Franzosa. We conclude with a brief discussion concerning transition systems and matrices. References for this latter material are [R, M1 and M2].

Recall that $\mathrm{CH}_{*}(S)$ is determined by an index pair. Thus, given a Morse decomposition $\mathscr{M}(S)=\{M(p) \mid p \in P\}$ to compute $C H_{*}(M(p))$ for each $p \in P$ we need to obtain an index pair. This can be done in a uniform manner which, as we shall see, allows one to make statements about the structure of $S$ from knowledge of $C H_{*}(M(p))$.

Definition 5.1. An index filtration of $\mathscr{M}(S)$ is a collection of compact sets, $\mathscr{N}(S,(P,>))=\mathscr{N}(S)=\{N(I) \mid I \in \mathscr{A}(P,>)\}$, satisfying

(a) for each $I \in \mathscr{A}(P,>),(N(I), N(\varnothing))$ is an index pair for $M(I)$.

(b) for each $I, J \in \mathscr{A}(P,>), N(I \cap J)=N(I) \cap N(J)$ and $N(I \cup J)=$ $N(I) \cup N(J)$.

If we let $J \in \mathscr{J}$, not necessarily an attracting interval, then there exists $I \in$ $\mathscr{A}$, such that $(I, J)$ is an adjacent pair of intervals and $I J \in \mathscr{A}$. One can now check that $(N(I J), N(I))$ is an index pair for $M(J)$. It is shown in [F1 or FM] that given a particular Morse decomposition $\mathscr{M}(S)=\{M(p) \mid p \in(P,>)\}$, one can always choose a fixed index filtration $\mathscr{N}(S)=\{N(I) \mid I \in \mathscr{A}(P,>)\}$.

As was mentioned earlier, the simplest nontrivial case of a Morse decomposition is an attractor repeller pair $\left(A, A^{*}\right)$. In this case, the index filtration is a nested triple of compact spaces $N_{0} \subset N_{1} \subset N_{2}$. The long exact sequence

$$
\stackrel{\partial}{\rightarrow} H_{k}\left(N_{1} / N_{0},\left[N_{0}\right]\right) \rightarrow H_{k}\left(N_{2} / N_{0},\left[N_{0}\right]\right) \rightarrow H_{k}\left(N_{2} / N_{1},\left[N_{1}\right]\right) \stackrel{\partial}{\rightarrow}
$$

induced by this triple defines the homology attractor repeller sequence

$$
\stackrel{\partial}{\rightarrow} \mathrm{CH}_{k}(A) \rightarrow C H_{k}(S) \rightarrow C H_{k}\left(A^{*}\right) \stackrel{\partial}{\rightarrow} \text {. }
$$

If $S=A \cup A^{*}$, the connection map $\partial$ is trivial, so the nontriviality of $\partial$ is a measure of the set of connecting orbits from $A^{*}$ to $A$.

For a general Morse decomposition $\mathscr{M}(S)=\{M(p) \mid p \in(P,>)\}$, every adjacent pair of intervals generates an index triple for the attractor repeller decomposition $(M(I), M(J))$ of $M(I J)$, and so generates a homology attractor repeller sequence. To understand how all of these induced homology sequences are related, we reformulate the sequence as follows.

In field coefficients, the sequence

$$
\stackrel{\partial}{\rightarrow} C H_{k}(M(I)) \rightarrow C H_{k}(M(I J)) \rightarrow C H_{k}(M(J)) \stackrel{\partial}{\rightarrow}
$$

defines a matrix $\Delta=\left(\begin{array}{ll}0 & \partial \\ 0 & 0\end{array}\right)$ on the vector space $C H_{*}(M(I)) \oplus C H_{*}(M(J))$. Since $\Delta^{2}=0,\left\{C H_{*}(M(I)) \oplus C H_{*}(M(J)), \Delta\right\}$ may be viewed as a chain complex. Further, if $C H_{*}(M(I))$ and $C H_{*}(M(J))$ are viewed as chain complexes with trivial boundary operators, then the sequence

$$
0 \rightarrow C H_{*}(M(I)) \rightarrow C H_{*}(M(I)) \oplus C H_{*}(M(J)) \rightarrow C H_{*}(M(J)) \rightarrow 0
$$


is a short exact sequence of chain complexes. Let $H_{*} \Delta(I J)$ denote the homology of $\left\{C H_{*}(M(I)) \oplus C H_{*}(M(J)), \Delta\right\}$. There is an isomorphism of homology sequences

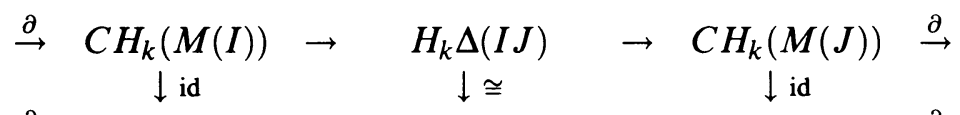

$$
\begin{aligned}
& \stackrel{\partial}{\rightarrow} C H_{k}(M(I)) \rightarrow C H_{k}(M(I J)) \rightarrow C H_{k}(M(J)) \stackrel{\partial}{\rightarrow}
\end{aligned}
$$

so the matrix $\Delta$ computes the homology attractor repeller sequence. Such a matrix is defined for every adjacent pair of intervals. In [F2] Franzosa shows that all of these matrices and their induced homology sequences may be derived from a single matrix known as a connection matrix.

To do so, fix a coefficient field $\mathscr{F}$ for all homology groups. For every interval $J$, let $C_{*} \Delta(J)=\bigoplus_{p \in J} C H_{*}(M(p))$. If $\Delta: C_{*} \Delta(P) \rightarrow C_{*} \Delta(P)$ is a matrix, then $\Delta_{p, q}$ is a linear map from $C H_{*}\left(M_{q}\right)$ to $C H_{*}\left(M_{p}\right) . \Delta$ is said to be strictly upper triangular if $q \ngtr p$ implies $\Delta_{p, q}=0$, and is said to be a boundary map if each $\Delta p, q$ is degree -1 and $\Delta^{2}=0$. If $\Delta: C_{*} \Delta(P) \rightarrow C_{*} \Delta(P)$ is a strictly upper triangular boundary map, and $I$ and $J$ are intervals in $P$, define

$$
\Delta(J, I): C_{*} \Delta(J) \rightarrow C_{*} \Delta(I)
$$

by $\Delta(J, I)=\left[\Delta_{p, q}\right]_{p \in I, p \in J}$. Let $\Delta(J, J)$ be denoted by $\Delta(J)$. It is easily checked that $\Delta(J)$ is an upper triangular boundary map on $C_{*} \Delta(J)$, i.e., $\Delta$ defines a chain complex $\left\{C_{*} \Delta(J), \Delta(J)\right\}$ for every interval $J$ in $P$. The homology of $\left\{C_{*} \Delta(J), \Delta(J)\right\}$ is denoted by $H_{*} \Delta(J)$. Notice that for $p \in$ $P,\left\{C_{*} \Delta(p), \Delta(p)\right\}=\left\{C H_{*} M(p), 0\right\}$ and thus $H_{*} \Delta(p)=C H_{*}(M(p))$. Furthermore, if $(I, J)$ is an adjacent pair of intervals, then the inclusions and projections of factors give a short exact sequence of chain complexes

$$
0 \rightarrow C_{*} \Delta(I) \rightarrow C_{*} \Delta(I J) \rightarrow C_{*} \Delta(J) \rightarrow 0
$$

and hence a long exact homology sequence

$$
\ldots \stackrel{\partial(J, I)}{\longrightarrow} H_{*} \Delta(I) \rightarrow H_{*} \Delta(I J) \rightarrow H_{*} \Delta(J) \stackrel{\partial(J, I)}{\longrightarrow} \cdots
$$

where $\partial(J, I)$ is the induced map on homology of $\Delta(J, I)$.

Definition 5.2 (Franzosa [F1]). A graded module braid isomorphism $\Psi$ is a collection of maps $\left\{\Psi(J): H_{*} \Delta(J) \rightarrow C H_{*}(M(J)) \mid J \in \mathscr{I}(P,>)\right\}$ such that $\Psi(p)=$ id for all $p \in P$, and such that for every adjacent pair of intervals $(I, J)$ the following diagram commutes:

$$
\begin{aligned}
& \ldots \stackrel{\partial(J, I)}{\longrightarrow} \begin{array}{c}
H_{*} \Delta(I) \\
\downarrow \Psi(I)
\end{array} \quad \rightarrow \quad \begin{array}{c}
H_{*} \Delta(I J) \\
\downarrow \Psi(I J)
\end{array} \quad \rightarrow \quad \begin{array}{c}
H_{*} \Delta(J) \\
\downarrow \Psi(J)
\end{array} \stackrel{\partial(J, I)}{\longrightarrow} \cdots \\
& \ldots \stackrel{\partial}{\longrightarrow} \mathrm{CH}_{k}(\mathrm{M}(I)) \rightarrow \mathrm{CH}_{k}(\mathrm{M}(I J)) \rightarrow \mathrm{CH}_{k}(\mathrm{M}(J)) \stackrel{\partial}{\longrightarrow} \ldots
\end{aligned}
$$

A simple induction argument shows that all of the $\Psi(j)$ 's are in fact isomorphisms.

Definition 5.3 (Franzosa [F2]). A connection matrix on $C_{*} \Delta(P)$ is a strictly upper triangular boundary map $\Delta: C_{*} \Delta(P) \rightarrow C_{*} \Delta(P)$ for which a graded module braid isomorphism exists.

Notice that for a given connection matrix $\Delta$ there may exist several graded module braid isomorphisms and, as has already been mentioned, for a given Morse decomposition, there may exist several connection matrices. 
Let $\mathscr{C}(\mathscr{M})=\mathscr{C}(\mathscr{M}(S),>)$ denote the set of connection matrices for a given Morse decomposition $\mathscr{M}(S)=\{M(p) \mid p \in(P,>)\}$. A fundamental theorem is

Theorem 5.4 (Franzosa [F2, Theorem 3.8]). $\mathscr{C}(\mathscr{M}) \neq \varnothing$.

To be useful for studying bifurcation problems the connection matrix must have well defined properties with regard to continuation. Adopting the same notation as was introduced in $\S 2$, let $\mathscr{C}\left(\mathscr{M}\left(S_{\lambda}\right)\right)=\mathscr{C}\left(\mathscr{M}\left(S_{\lambda}\right),>_{\lambda}\right)$ denote the set of connection matrices for $M\left(S_{\lambda}\right)=\left\{M_{\lambda}(p) \mid p \in(P,>)\right\}$ under the flow $\varphi_{\lambda}$.

Theorem 5.5 (Franzosa [F3, Theorem 5.7]). Let $\lambda_{0} \in \Lambda$, then there exists a neighborhood $U$ of $\lambda_{0}$ such that $\mathscr{C}\left(\mathscr{M}\left(S_{\lambda}\right)\right) \subset \mathscr{C}\left(\mathscr{M}\left(S_{\lambda_{0}}\right)\right)$ for all $\lambda \in U$.

Theorem 5.6 (Franzosa [F3, Theorem 5.7]). Let $K$ be a connected subset of $\Lambda$ and let $\lambda, \mu \in K$. Let $S \subset X \times K$ be an isolated invariant set with Morse decomposition $\mathscr{M}(S)=\{M(p) \mid P \in(P,>)\}$, then

$$
\mathscr{C}\left(\mathscr{M}\left(S_{\lambda}\right),>_{\lambda}\right)=\mathscr{C}\left(\mathscr{M}\left(S_{\mu}\right),>_{\mu}\right) .
$$

An alternative statement of Theorems 5.5 and 5.6 is that the set of connection matrices is upper semicontinuous on $\Lambda$ and is constant over those subsets of $\Lambda$ for which the flow defined order continues.

Proposition 5.7. Let $>_{1}$ and $>_{2}$ be admissible orderings for $\mathscr{M}(S)$ and assume that $>_{1}$ is an extension of $>_{2}$, i.e., $p>_{2} q$ implies $p>_{1} q$. Then

$$
\mathscr{C}\left(\mathscr{M}(S),>_{2}\right) \subset \mathscr{C}\left(\mathscr{M}(S),>_{1}\right) \text {. }
$$

Obviously, as defined, the best that the connection matrix can do is to give information about the structure of an invariant set in the neighborhood of a fixed parameter value. Equally clear is that our discussion of connected simple systems involved studying bifurcations over a large set of parameter values. Therefore, we now turn to a discussion of transition matrices. These matrices are used to measure the "difference" between connection matrices at different parameter values and thereby to give information about the existence of global bifurcations such as the existence of heteroclinic orbits. For the sake of clarity we shall restrict our discussion to a parameter space $\Lambda=[-1,2] \subset \mathbf{R}$, to a total space $X=\mathbf{R}^{n}$, and to flows $\varphi_{\lambda}$ generated by the ordinary differential equation $x^{\prime}=f(x, \lambda)$. Using the same notation as in $\S 2$, this defines a parameter flow $\vartheta: \mathbf{R}^{n} \times[-1,2] \times \mathbf{R} \rightarrow \mathbf{R}^{n} \times[-1,2]$. We shall define $\vartheta_{n}$ to be the local flow defined by $x^{\prime}=f(x, \lambda), \lambda^{\prime}=\frac{1}{m} \lambda(\lambda-1)$. Notice that for $\lambda=0$ and $\lambda=1$ we recover the flows $\varphi_{0}$ and $\varphi_{1}$ on the subspaces $\mathbf{R}^{n} \times\{0\}$ and $\mathbf{R}^{n} \times\{1\}$. Furthermore, as $m \rightarrow \infty, \vartheta_{m} \rightarrow \vartheta$, thus by studying the sequence of flows $\left\{\vartheta_{m}\right\}$ one can hope to recover information about the flows $\varphi_{\lambda}$ for $\lambda \in(0,1)$.

Again using the notation of $\S 2$, let $S$ be an isolated invariant set for $\vartheta$ with a Morse decomposition $\mathscr{M}(S)=\{M(p) \mid p \in(P,>)\}$. Then, we have isolated invariant sets and Morse decompositions $\mathscr{M}\left(S_{0}\right)=\left\{M_{0}(p) \mid p \in(P,>)\right\}$ and $\mathscr{M}\left(S_{1}\right)=\left\{M_{1}(p) \mid p \in(P,>)\right\}$. This in turn leads to connection matrices $\Delta_{0}$ and $\Delta_{1}$, respectively. For $m$ sufficiently large, $\vartheta_{m}$ has a Morse decomposition $\mathscr{M}\left(S_{m}\right)=\left\{M_{i}(p) \mid i=0,1\right.$ and $\left.p \in(P,>)\right\}$ with a corresponding connection matrix

$$
\left[\begin{array}{cc}
\Delta_{0} & T_{m} \\
0 & \Delta_{1}^{+}
\end{array}\right]
$$


where $\Delta_{1}^{+}$is a degree +1 conjugation of $\Delta_{1}$ (this conjugation is necessary because at $\lambda=1$ we have increased the dimension of the unstable manifold by one which introduces a suspension to the Conley index). Since there are only a finite number of possible matrices $T_{m}$, the sequence $\left\{T_{m}\right\}$ must have a convergent subsequence. Let $T$ denote the limit of such a convergent subsequence, then $T$ is called a transition matrix. Ignoring the +1 conjugation on $\Delta_{1}$ one obtains the following result (see [R, M1, and M2].)

Theorem 5.8. A transition matrix from $\lambda=1$ to $\lambda=0$ satisfies the following properties:

(i) $\Delta_{0} T+T \Delta_{1}=0$ ( $T$ is now a degree 0 map),

(ii) $T$ is an isomorphism,

(iii) $T$ is upper triangular with respect to $>$.

(iv) if $T(p, q): H(p) \rightarrow H(q)$ is nonzero, then there exists a finite sequence $1>\lambda_{1}>\lambda_{2}>\cdots>\lambda_{k}>0$ and corresponding $p_{i} \in P$ such that $p_{i}>\lambda_{i} P_{i+1}$ where $>_{\lambda_{i}}$ is the flow defined order under $\varphi_{\lambda_{i}}$.

Notice that (i) indicates that if two connection matrices are related by an order preserving continuation of the Morse decomposition then they are conjugate, i.e., $\Delta_{1}=-T^{-1} \Delta_{0} T$.

\section{TRANSITION MATRICES}

As was indicated earlier to detect global bifurcations one needs to be able to commpare connection matrices at different parameter values. In $\S 5$, this was done via transition matrices. This approach is, if nothing else, esthetically unpleasing since it involves introducing an artificial parameter flow, which is in the end ignored. A more serious problem is that it requires the parameter space to be compact. An alternative is to try to begin with the algebraic equation

$$
\Delta_{0} T+T \Delta_{1}=0 \text {. }
$$

This has problems in that $T=0$ is an obvious solution. Furthermore, even if one were to require that $T$ be an isomorphism, $T$ need not be unique. In particular, if $\Delta_{i}=0$ then any isomorphism satisfies (6.1).

In this section we indicate an alternative definition of transition matrices, using the map $T_{\lambda \mu}$ defined in $\S 3$. We will develop this definition only in a restricted setting, but we believe it can be extended to provide a general definition of transition matrices.

We begin with $S$, an isolated invariant set, for $\vartheta$ with a Morse decoinposition $\mathscr{M}(S)=\{M(p) \mid p \in(P,>)\}$. Then, we have isolated invariant sets and Morse decompositions $\mathscr{M}\left(S_{\lambda}\right)=\left\{M_{\lambda}(p) \mid p \in(P,>)\right\}$ and $\mathscr{M}\left(S_{\mu}\right)=\left\{M_{\mu}(p) \mid p \in(P,>)\right\}$. This in turn leads to connection matrices $\Delta_{\lambda}$ and $\Delta_{\mu}$, respectively. Let $(J, K) \in \mathscr{I}_{2}(>)$ with the property that $M_{a}(J K)=\bigcup_{p \in J K} M_{a}(p)$ for $a \in \Lambda$. In this case, $\Delta(J K)=0$ and hence

$$
H \Delta(J K)=\bigoplus_{p \in J K} H(p)
$$

Now by definition of the connection matrix, we have the following commutative diagram.

$$
\begin{array}{ccccccc}
0 \rightarrow \quad H \Delta(J, a) & \rightarrow & H \Delta(J, a) \oplus H \Delta(K, a) & \rightarrow & H \Delta(K, a) & \rightarrow 0 \\
\Psi_{a}(J) \downarrow & & \Psi_{a}(J K) \downarrow & & \Psi_{a}(K) \downarrow & \\
0 \rightarrow \quad C H(J, a) & \rightarrow & C H(J K, a) & \rightarrow & C H(K, a) & \rightarrow 0
\end{array}
$$


where $a=\lambda$ or $\mu$, and the $\Psi(\cdot)$ are the isomorphisms of some graded module braid isomorphism between the graded module braid generated by $\bigoplus_{p \in J K} H(p)$ and the homology index braid.

In the definition of a connection matrix, the only restriction on the $\Psi$ 's is that they be isomorphisms and that they make (6.4) commute. Our idea is to use the methods of $\S 3$ to define $\Psi$. Let $\Phi(J, a)$ be as in $\S 3$. Define

$$
\Psi(J, \lambda)=C H_{*} \Phi(J, \lambda)=\Phi(J, \lambda)
$$

(the latter equality is obtained by abuse of notation). Thus the $C H_{*}$ functor gives the following diagram.

$$
\begin{array}{ccc}
\bigoplus_{p \in J K} H \Delta(p, \lambda) \stackrel{E_{\lambda \mu}(J K), T_{\lambda \mu}(J K)}{\longrightarrow} & \bigoplus_{p \in J K} H \Delta(p, \mu) \\
\Psi_{\lambda}(J K) \downarrow & \Psi_{\mu}(J K) \downarrow \\
C H(J K, \lambda) & \stackrel{F_{\lambda \mu}(J K)}{\longrightarrow} & C H(J K, \mu)
\end{array}
$$

Notice that this is exactly the homology version of (3.1) and hence Proposition 3.5 applies. This leads us to the following theorem.

Theorem 6.1. Let $\Lambda=[0,1]$ and let $\mathscr{M}(S)=\{M(p) \mid p \in(P,>)\}$ be a Morse decomposition for the parameterized flow. Assume that $S_{\lambda}=\bigcup_{p \in P} M_{\lambda}(p)$ for $\lambda=0,1$. Then $T_{10}(P)=T: \bigoplus_{p \in P} H \Delta(p, 1) \rightarrow \bigoplus_{p \in P} H \Delta(p, 0)$ satisfies the following properties:

(i) $\Delta_{0} T+T \Delta_{1}=0$,

(ii) $T$ is an isomorphism,

(iii) $T$ is upper triangular with respect to $>$,

(iv) if $T(p, q): H(p, 1) \rightarrow H(q, 0)$ is nonzero, then there exists a finite sequence $1>\lambda_{1} \geq \lambda_{2} \geq \cdots \geq \lambda_{k} \geq 0$ and corresponding $p_{i} \in P$ such that $p_{1}=p, p_{k}=q$, and $p_{i}>_{\lambda_{i}} p_{i+1}$ where $>_{\lambda_{i}}$ is the flow defined order under $\varphi_{\lambda_{i}}$. Proof. (i) follows since $\Delta_{\lambda}=0$. (ii) and (iii) follow from Proposition 3.5. Thus we only need prove (iv). The existence of $\left\{\lambda_{i}\right\}$ and the corresponding $\left\{p_{i}\right\}$ follows from (iii). Thus one only needs to show that $\lambda_{i} \geq \lambda_{i+1}$. However, this follows from a simple proof by induction (using the number of elements between $p$ and $q$ ) and use of the product formula of Proposition 3.1(ii).

Because of Theorem 6.1, we shall refer to $T_{\lambda \mu}$ as a transition matrix. To give a concrete description of these ideas we shall consider two examples concerning connection matrices with the parameter space $\Lambda=[0,1]$. Both of these examples are based on the flow described in Figure 3. Let the critical points $x$ and $y$ be Morse sets and let $S_{a}$ and $S_{b}$ be determined by the flow at parameter values $\lambda=0$ and 1 , respectively. Using $\mathbf{Z}_{2}$ coefficients we leave it to the reader to check the following simple facts:

$$
\begin{aligned}
& C H_{*}(x) \approx\left(0, \mathbf{Z}_{2}, 0,0, \ldots\right), \\
& C H_{*}(y) \approx\left(0, \mathbf{Z}_{2}, 0,0, \ldots\right), \\
& C H_{*}(S) \approx\left(0, \mathbf{Z}_{2} \oplus \mathbf{Z}_{2}, 0,0, \ldots\right), \\
& \Delta_{0}=\Delta_{1}=0, \text { and } \\
& T_{10}: C H_{*}(y) \oplus C H_{*}(x) \rightarrow C H_{*}(y) \oplus C H_{*}(x) \text { is given by } T_{10}=\left(\begin{array}{ll}
1 & 1 \\
0 & 1
\end{array}\right) .
\end{aligned}
$$

Example 6.2. We consider the parameterized flow described by Figure 8. In particular we assume that $3>2>1$ is an admissible order for $\mathscr{M}(S)=$ 

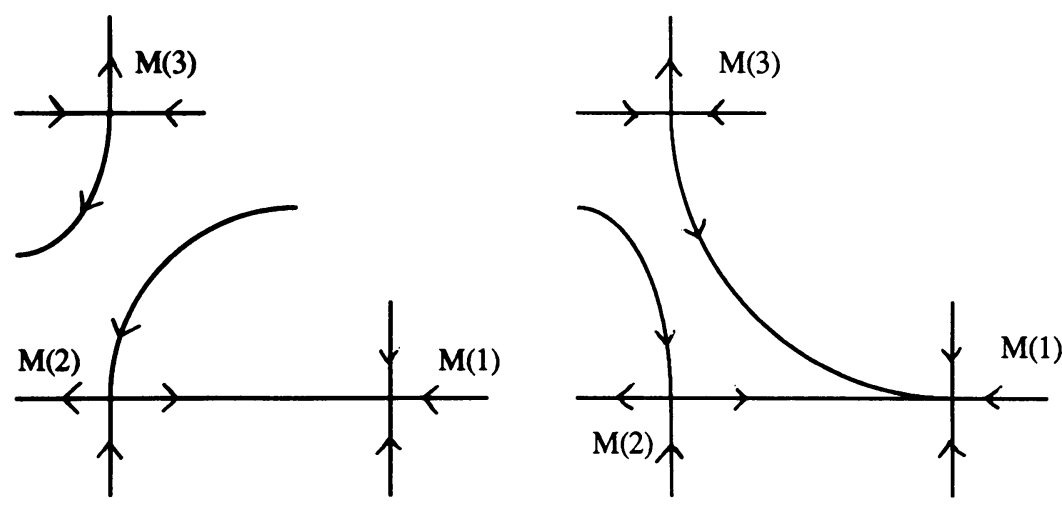

FigURE 8

$\{M(p) \mid p=1,2,3\}$ the Morse decomposition of the parameterized flow. Notice that this example does not fall into the setting of $S=\bigcup_{p \in P} M(p)$. However, $T$ must be a degree 0 map and $T(p, p)=$ id therefore the only undetermined entry is $T(3,2)$. Furthermore, $M(32)=M(3) \cup M(2)$, and hence, the above remarks apply.

In [F2] it is shown that $\Delta_{\lambda}: C H_{*}(1) \oplus C H_{*}(2) \oplus C H_{*}(3) \rightarrow C H_{*}(1) \oplus C H_{*}(2) \oplus$ $\mathrm{CH}_{*}(3)$ is given by

$$
\Delta_{0}=\left(\begin{array}{lll}
0 & 1 & 0 \\
0 & 0 & 0 \\
0 & 0 & 0
\end{array}\right) \text { and } \Delta_{1}=\left(\begin{array}{lll}
0 & 1 & 1 \\
0 & 0 & 0 \\
0 & 0 & 0
\end{array}\right) .
$$

This implies, by Theorem 5.7, that there exists $\lambda^{*} \in(0,1)$ such that both $\Delta_{0}$ and $\Delta_{1}$ are connection matrices for $M\left(S_{\lambda^{*}}\right)$. Either by consulting [F2] or by a simple calculation, the reader can establish that the connection matrices at $\lambda$ and $\mu$ are equal if and only if $\Psi_{\lambda}(32)=\Psi_{\mu}(32)$. However $\Psi(32)$ is just $\Phi_{\lambda}(x y)$ from the example of Figure 3. Furthermore, if we compute the transition matrix $T_{10}$ we get

$$
T_{10}=\left(\begin{array}{lll}
1 & 0 & 0 \\
0 & 1 & 1 \\
0 & 0 & 1
\end{array}\right)
$$

i.e., the connected simple systems changed and hence we detect a change in the maps $\Phi_{0}(x y)$ and $\Phi_{1}(x y)$. The point to be made here is that connection matrices can be distinguished from one another via a flow defined map. Also, the nonuniqueness of connection matrices at $\lambda=\lambda^{*}$ occurs because $\lambda^{*} \notin \Lambda$, and hence, the map $\Phi(x y)$ cannot be defined.

Example 6.3. Now consider the one parameter family of flows indicated by Figure 9. In this case $\Delta_{0}=\Delta_{1}=\Delta_{\lambda}=0$ for $\lambda \in(0,1)$. Just as before, we have

$$
T_{10}=\left(\begin{array}{ccc}
1 & 0 & 0 \\
0 & 1 & 1 \\
0 & 0 & 1
\end{array}\right) \text {. }
$$

The point is that there exists some $\lambda^{*} \in(0,1)$ for which an $M(3) \in M(2)$ connection occurs, i.e., a global bifurcation occurs. However, the connection 

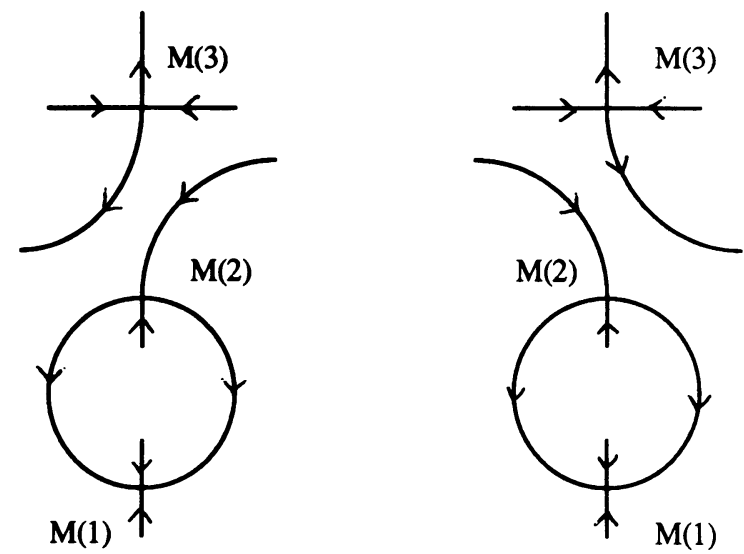

FIGURE 9

matrix does not detect this and in particular the set of connection matrices is unique throughout the parameter space. Nevertheless, one can, by way of the connected simple systems, associate different graded module braid isomorphisms to the connection matrices for $\lambda \in\left[0, \lambda^{*}\right)$ and $\lambda \in\left(\lambda^{*}, 1\right]$. Thus, the "nonuniqueness" at the bifurcation parameter, appears not in the connection matrix $\Delta_{\lambda}$, but in the isomorphism $\Psi(32)$, which cannot be uniquely defined.

\section{REFERENCES}

[CDT] S.-N. Chow, B. Deng and D. Terman, The bifurcation of a homoclinic orbit-a topological approach, preprint CDSNS90-12.

[C] C. Conley, Isolated invariant sets and the Morse index, CBMS Regional Conf. Ser. in Math. no. 38, Amer. Math. Soc., Providence, R.I., 1978.

[F1] R. Franzosa, Index filtrations and the homology index braid for partially ordered Morse decompositions, Trans. Amer. Math. Soc. 298 (1986), 193-213.

[F2] _ The connection matrix theory for Morse decompositions, Trans. Amer. Math. Soc. 311 (1989), 561-592.

[F3] _ The continuation theory for Morse decompositions and connection matrices, Trans. Amer. Math. Soc. 310 (1988), 781-803.

[FM] R. Franzosa and K. Mischaikow, The connection matrix theory for semiflows on (not necessarily locally compact) metric spaces, J. Differential Equations 71 (2) (1988), 270-287.

[K] H. Kokubu, Homoclinic and heteroclinic bifurcations of vector fields, Japan J. Appl. Math. 5 (1988), 455-501.

[Ku1] H. Kurland, The Morse index of an isolated invariant set is a connected simple system, $\mathrm{J}$. Differential Equations 42 (1981).

[Ku2] _ Homotopy invariants of repeller attractor paris, I. The Puppe sequence of an $R-A$ pair, J. Differential Equations 46 (1982).

[Ku3] _ Homotopy invariants of repeller attractor pairs, II. Continuation of $R-A$ pairs, $\mathrm{J}$. Differential Equations 49 (1983).

[M1] K. Mischaikow, Existence of generalized homoclinic orbits for one-parameter families of flows, Proc. Amer. Math. Soc. 103 (1989), 59-68.

[M2] _-, Transition systems, Proc. Roy. Soc. Edinburgh Sect. A 112 (1989), 155-175.

[Mo] R. Moeckel, Morse decompositions and connection matrices, Ergodic Theory Dynamical Systems 8 (1988), 227-250.

[R] J. Reineck, Connecting orbits in one-parameter families of flows, Ergodic Theory Dynamical Systems 8 (1988), 359-374. 
[Ry] K. Rybakowski, The homotopy index and partial differential equations, Universitext, Springer-Verlag, 1987.

[S] D. Salamon, Connected simple systems and the Conley index of isolated invariant sets, Trans. Amer. Math. Soc. 291 (1985).

[Sm] J. Smoller, Shock waves and reaction diffusion equations, Springer-Verlag, Berlin and New York, 1983.

Department of Mathematical Sciences, University of Cincinnati, Cincinnati, Ohio 45221

Center for Dynamical Systems and Nonlinear Studies, Georgia Institute of TechNOlogy, Atlanta, Georgia 30332 